\title{
Misalignment of Magnetic Fields, Outflows and Discs in Star-forming Clouds
}

\author{
Masahiro N. Machida, ${ }^{1 \star}$ Shingo Hirano ${ }^{1}$ and Hideyuki Kitta ${ }^{1}$ \\ Department of Earth and Planetary Sciences, Faculty of Sciences, Kyushu University, Fukuoka, Fukuoka 819-0395, Japan
}

\begin{abstract}
Using resistive magnetohydrodynamics simulations, the propagation of protostellar jets, the formation of circumstellar discs and the configuration of magnetic fields are investigated from the prestellar cloud phase until $\sim 500 \mathrm{yr}$ after protostar formation. As the initial state, we prepare magnetized rotating clouds, in which the rotation axis is misaligned with the global magnetic field by an angle $\theta_{0}$. We calculate the cloud evolution for nine models with different $\theta_{0}\left(=0,5,10,30,45,60,80,85,90^{\circ}\right)$. Our simulations show that there is no significant difference in the physical quantities of the protostellar jet, such as the mass and momentum, among the models except for the model with $\theta_{0}=90^{\circ}$. On the other hand, the directions of the jet, disc normal and magnetic field are never aligned with each other during the early phase of star formation except for the model with $\theta_{0}=0^{\circ}$. Even when the rotation axis of the prestellar cloud is slightly inclined to the global magnetic field, the directions of the jet, disc normal and local magnetic field differ considerably, and they randomly change over time. Our results indicate that it is very difficult to extract any information from the observations of the directions of the outflow, disc and magnetic field at the scale of $\lesssim 1000 \mathrm{au}$. Thus, we cannot use such observations to derive any restrictions on the star formation process.
\end{abstract}

Key words: MHD - stars: formation - stars: protostars - stars: magnetic field - stars: winds, outflows

^ E-mail: machida.masahiro.018@m.kyushu-u.ac.jp (MNM) 


\section{INTRODUCTION}

Magnetic fields and rotation play significant roles in the star formation process. The rotation of a prestellar cloud results in the formation of a Keplerian disc, which is the host of planet formation (Havashi et al. 1985). The presence of a magnetic field leads to the emergence of protostellar jets, which transfer an excess angular momentum and determine the star formation efficiency and the final stellar mass (Nakano et al. 1995). Since magnetic fields and angular momentum are vectors, their directions should be considered. For example, the efficiency of magnetic braking depends on the mutual angle between the magnetic and angular momentum vectors of the prestellar cloud (Mouschovias \& Paleologou 1979, 1980a). In addition, the directions of the magnetic and angular momentum vectors influence the disc formation process (Matsumoto \& Tomisaka 2004; Hennebelle \& Ciardi 2009; Tsukamoto et al. 2018).

In many theoretical studies, the magnetic fields are assumed to be parallel to the rotation axis for simplicity (Larson 2003, and references therein). In numerical simulations, researchers have calculated the evolution of rotating magnetized clouds assuming that the rotation axis is parallel to the global magnetic field (Tomisaka 2002; Machida et al. 2004; Banerjee \& Pudritz 2006; Tomida et al. 2013; Bate et al. 2014; Vaytet et al. 2018).

Matsumoto \& Tomisaka (2004) first investigated the misalignment case, in which the rotation axis of the prestellar cloud is inclined to the global magnetic field. They showed that the directions of the local magnetic field and disc normal (or local angular vector) tended to converge. Although they did not show perfect alignment, the outflow direction roughly agrees with the direction of the local magnetic field, not the direction of the global magnetic field.

Hennebelle \& Ciardi (2009) also investigated cloud collapse when the global magnetic field is not parallel to the cloud rotation axis. Although they did not comment on the outflow direction, the outflow seems not to be aligned with the initial magnetic field. In these studies, the calculations were executed in the ideal magnetohydrodynamics (MHD) approximation. However, in reality, dissipation of the magnetic field can significantly reduce the efficiency of magnetic braking (Machida et al. 2011). Since the direction of the rotationally supported disc (or the disc normal direction) is changed by magnetic braking (Joos et al. 2012), and the disc drives protostellar jets, the magnetic dissipation should be considered when investigating the directions of the disc and jets. 
Using non-ideal MHD simulations, Masson et al. (2016) and Tsukamoto et al. (2018) investigated cloud evolution for a rotation axis that was not aligned with the global magnetic field (i.e. the misalignment case) and discussed the size growth of the circumstellar disc. They calculated the gas collapsing phase just before and after protostar formation. Thus, we cannot know the directions of the evolved protostellar jet and disc from their simulations. Wurster \& Bate (2019) investigated disc formation and fragmentation with the sink particle technique in non-ideal MHD simulations. They were able to calculate the long-term evolution during the main accretion phase, while the high-speed jet does not emerge in their simulations due to adopting a large sink radius of 1 au.

Hull et al. (2013) noted that observed magnetic fields at a large scale are not aligned with the outflow directions. The speed of observed outflows exceeds $10 \mathrm{~km} \mathrm{~s}^{-1}$ with a scale of $\sim 1000 \mathrm{au}$. In contrast, in many simulations, the outflow speed does not reach $\sim 10 \mathrm{~km} \mathrm{~s}^{-1}$ (Matsumoto \& Tomisaka 2004; Machida et al. 2005; Hennebelle \& Ciardi 2009; Seifried et al. 2012; Wurster \& Bate 2019). Thus, the outflows in past simulations are much slower than those in observations. Only low-velocity outflows appear in the simulations either because the spatial resolution of the simulations is very low or because the time integration is not sufficient to evolve high-speed flows. Whatever the reason, there is currently a significant gap between past simulations and observations with regard to the speed of outflows.

Recent developments in super computers enable the calculation of long term cloud evolution with a high spatial resolution. Recent studies have calculated an outflow reaching $\sim 1000$ au with a speed of $\gtrsim 100 \mathrm{~km} \mathrm{~s}^{-1}$, in which a protostar can be resolved with a sufficiently high spatial resolution (Machida et al. 2014; Machida \& Basu 2019). In addition, Hirano \& Machida (2019, hereafter Paper I) showed that the outflow directions differ for different speeds, so that the low-velocity component is misaligned with the high-velocity component, which was confirmed in a recent observation (Matsushita et al. 2019). We can now reasonably compare simulations with observations.

The purpose of this study is to verify the star formation process in numerical simulations compared with observations. We focus on the directions of the outflow, disc and magnetic field in the early phase of star formation. Our paper is structured as follows. We present the initial conditions and numerical settings in $\S 2$. The simulation results are shown in $\S 3$. We quantitatively discuss differences between the alignment and misalignment cases in $\S 4$. We summarize our results in $\S 5$. 


\section{INITIAL CONDITIONS AND NUMERICAL SETTINGS}

The initial conditions and numerical settings in this study are the same as those in Paper I, so we only briefly describe them here. As the initial state, we adopt a Bonnor-Ebert sphere with a central density of $6 \times 10^{5} \mathrm{~cm}^{-3}$ (or $\left.2.4 \times 10^{-18} \mathrm{~g} \mathrm{~cm}^{-3}\right)$ and an isothermal temperature of $10 \mathrm{~K}$. To promote cloud contraction, the density of the Bonnor-Ebert sphere is uniformly enhanced by a factor of 1.8 (Machida \& Hosokawa 2013). The initial cloud has a radius of $1.18 \times 10^{4}$ au and a mass of $2.1 M_{\odot}$. A rigid rotation of $\Omega_{0}=1.4 \times 10^{-13} \mathrm{~s}^{-1}$ is set inside the initial cloud, while a uniform magnetic field of $B_{0}=5.7 \times 10^{-5} \mathrm{G}$ is imposed over the whole computational domain. The ratios of the thermal $\left(\alpha_{0}\right)$ and rotational $\left(\beta_{0}\right)$ energies to the gravitational energy are $\alpha_{0}=0.39$ and $\beta_{0}=0.026$. The mass-to-flux ratio normalized by the critical value $\left(2 \pi G^{1 / 2}\right)^{-1}$ is $\mu_{0}=1.2$. These non-dimensional parameters $\left(\alpha_{0}, \beta_{0}\right.$ and $\left.\mu_{0}\right)$ are the same as those adopted in Machida et al. (2014), Machida \& Basu (2019) and Paper I.

We define the initial mutual angle $\theta_{0}$ between the rotation axis of the initial cloud and the global magnetic field, and use it as a parameter. Each model is characterized only by the parameter $\theta_{0}$. We take nine different initial angles $\theta_{0}=0,5,10,30,45,60,80,85$ and $90^{\circ}$ and execute calculations for these nine models. The model names and parameters are summarized in Table 1 .

To calculate the cloud evolution for these models, we use our nested grid code, in which rectangular grids are nested (for details, see Machida et al. 2004, 2005, 2007, 2011, 2014). Each grid is composed of $(i, j, k)=(64,64,64)$ cells. In Cartesian coordinates, the uniform magnetic fields are set along the $z$-axis, while the rotation axis is inclined from the $z$-axis to the $x$-axis by the angle $\theta_{0}$.

Each grid level is described by $l$, and the minimum and maximum grid levels are set to $l=1$, which corresponds to the coarsest grid, and $l=20$, which corresponds to the finest grid, respectively. The grid size and cell width halve with each increment of grid level. The grid size $L(l)$ and cell width $h(l)$ of the coarsest grid $(l=1)$ are $L(1)=3.78 \times 10^{5}$ au and $h(1)=591 \mathrm{au}$, while the finest or maximum grid $(l=20)$ has $L(20)=0.72$ au and $h(20)=0.011 \mathrm{au}$.

The initial cloud is immersed in the fourth level grid $(l=4)$, whose grid size is equal to twice the Bonner-Ebert radius $\left(2.36 \times 10^{4} \mathrm{au}\right)$. A uniform density of $n_{\text {ISM }}=1.35 \times 10^{4} \mathrm{~cm}^{-3}$ is set for the exterior of the initial cloud, and the numerical boundary is set to the surface of the $l=1$ grid. Thus, outside the initial cloud, the interstellar medium is distributed in 
the range of $1.18 \times 10^{4} \mathrm{au}<r<1.89 \times 10^{5} \mathrm{au}$. A large area of interstellar space is used to prevent any artificial reflection of the Alfvén wave at the boundary (Machida \& Hosokawa 2013). A finer grid is automatically generated to resolve the Jeans wavelength, of at least 16 cells.

The basic equations used in this study are as follows:

$$
\begin{gathered}
\frac{\partial \rho}{\partial t}+\nabla \cdot(\rho \boldsymbol{v})=0 \\
\rho \frac{\partial \boldsymbol{v}}{\partial t}+\rho(\boldsymbol{v} \cdot \nabla) \boldsymbol{v}=-\nabla P-\frac{1}{4 \pi} \boldsymbol{B} \times(\nabla \times \boldsymbol{B})-\rho \nabla \phi \\
\frac{\partial \boldsymbol{B}}{\partial t}=\nabla \times\left[\boldsymbol{v} \times \boldsymbol{B}-\eta_{O}(\nabla \times \boldsymbol{B})\right], \\
\nabla^{2} \phi=4 \pi G \rho
\end{gathered}
$$

where $\rho, \boldsymbol{v}, P, \boldsymbol{B}, \eta_{O}$ and $\phi$ denote the density, velocity, pressure, magnetic flux density, Ohmic resistivity and gravitational potential, respectively. We use the fitting fomura of the Ohmic resistivity $\eta_{O}$, which was derived in Machida et al. (2007) and is described as

$$
\eta_{O}=\frac{740}{X_{e}} \sqrt{\frac{T}{10 \mathrm{~K}}}\left[1-\tanh \left(\frac{n}{10^{15} \mathrm{~cm}^{-3}}\right)\right] \mathrm{cm}^{2} \mathrm{~s}^{-1}
$$

where $T$ and $n$ are the gas temperature and the number density of molecular hydrogen, and $X_{e}$ is the ionization degree of the gas which describes as

$$
X_{e}=5.7 \times 10^{-4}\left(\frac{n}{\mathrm{~cm}^{-3}}\right)^{-1} \text {. }
$$

Further information about equations (5) and (6) can be referred to Nakano et al. (2002). For the gas pressure and temperature, the barotropic equation of state $P \propto \rho^{\Gamma}\left(\right.$ or $\left.T \propto \rho^{\Gamma-1}\right)$ is used (for detals, see Machida et al. 2007, 2018). The polytropic exponent $\Gamma$ is set as

$$
\Gamma=\left\{\begin{array}{lll}
1 & \text { for } & \rho<8.0 \times 10^{-14} \mathrm{~g} \mathrm{~cm}^{-3} \\
5 / 3 & \text { for } & 8.0 \times 10^{-14} \mathrm{~g} \mathrm{~cm}^{-3}<\rho<1.2 \times 10^{-10} \mathrm{~g} \mathrm{~cm}^{-3} \\
7 / 5 & \text { for } & 1.2 \times 10^{-10} \mathrm{~g} \mathrm{~cm}^{-3}<\rho<4.0 \times 10^{-9} \mathrm{~g} \mathrm{~cm}^{-3} \\
1.1 & \text { for } & 4.0 \times 10^{-9} \mathrm{~g} \mathrm{~cm}^{-3}<\rho<1.2 \times 10^{-5} \mathrm{~g} \mathrm{~cm}^{-3} \\
2 & \text { for } & \rho>1.2 \times 10^{-5} \mathrm{~g} \mathrm{~cm}^{-3}
\end{array}\right.
$$

The equation of state in the range $\rho<1.2 \times 10^{-5} \mathrm{~g} \mathrm{~cm}^{-3}$ approximately reproduces the thermal evolution shown in past studies (Larson 1969; Masunaga \& Inutsuka 2000; Tomida et al. 2013). On the other hand, in order to realize a long-term integration, a stiff equation of state (or larger $\Gamma$ ) is adopted in the range of $\rho>1.2 \times 10^{-5} \mathrm{~g} \mathrm{~cm}^{-3}$, which also mimics the protostar having a size of $\ll 10$ solar radius (for details, see Machida et al. 2014; Machida \& Nakamura 2015; Machida \& Basu 2019; Hirano \& Machida 2019). Thus, the gas temperature is only a (C) 2019 RAS, MNRAS 000, 11?? 
function of density with the barotropic approximation, and the change in temerature due to such as shock heating and protostellar irradiation is ignored. Since we only focus on the very early phase of star formation (see \$3), the heating (and cooling) effects are expected to not be significant. However, we need to carefully consider the gas temperature because it is related to the ionization degree and Ohmic resistivity which detemine the configuration of magnetic field in a small scale. We will focus on the effect of heating and cooling on the magnetic configuration in future studies. The numerical methods and settings are fully described in our previous studies (Machida et al. 2004, 2005, 2006; Machida \& Hosokawa 2013; Machida et al. 2014, 2018; Machida \& Basu 2019; Hirano \& Machida 2019).

\section{RESULTS}

We calculated the cloud evolution from the prestellar cloud stage until about $500 \mathrm{yr}$ after protostar formation for the nine models. This section describes the cloud evolutions for the models T00 and T90 and then presents the results for the models T05 to T85.

\subsection{Rotation Axis Parallel to Magnetic Field: $\theta_{0}=0^{\circ}$}

Figure 1 shows the density and velocity distributions on the $x=0, y=0$ and $z=0$ cutting planes at $t_{\mathrm{ps}}=462.2 \mathrm{yr}$ for model T00, where $t_{\mathrm{ps}}$ is the elapsed time after protostar formation. The protostar has a mass of $\sim 0.033 M_{\odot}$ at this epoch. 1 For this model, both the initial magnetic field and initial rotation vectors are set to be parallel to the $z$-axis. As typically seen in past simulations (Bate 1998; Machida et al. 2014; Tomida et al. 2015), a rotationally supported disc forms around the protostar (Fig. 1k). The disc has a size of $\sim 5 \mathrm{au}$ (Figs. 11 d and e). As also seen in past studies (Tomisaka 2002; Banerjee \& Pudritz 2006; Machida et al. 2006), both low- and high-velocity flows appear above and below the disc. The low-velocity flow has a wide opening angle, while the high-velocity component has a narrow structure (Figs. 1 $a, b, d$ and $e$ ). We can confirm that the high-velocity flow is driven near the protostar in Figures $1 g$ and $h$. The flow speed exceeds $20 \mathrm{~km} \mathrm{~s}^{-1}$ near the protostar. In Figures $1 a$ and $b$, we can also confirm a thin pseudo disc that surrounds the rotationally supported disc around the protostar, as also seen in observations (Riaz et al. 2019; Lee et al. 2019). The outflow reaches $\sim 500$ au by this epoch. The knotty structure seen in the high velocity component (Figs. 1 $a$ and $b$ ) is caused by episodic mass ejection

1 We define the protostar as the region where the number density exceeds $n>10^{1} 8 \mathrm{~cm}^{-3}$ as described in Paper I. 
(Machida 2014; Machida \& Basu 2019). The pseudo disc, rotationally supported disc and low- and high-velocity outflows during the early stage of the star formation are typically seen in both simulations and observations.

Figure 2 shows three-dimensional views of the magnetic field lines, outflows and disc for model T00 with different spatial scales. In the large scale, we can confirm a clear hourglass structure of the magnetic field lines (Fig. 2 $2 a$ ). The hourglass configuration has also been confirmed in observations (Girart et al. 2006, 2009). As the cloud collapses, the magnetic field lines are pulled toward the center of the cloud. Then, the magnetic field lines converge to the center and exhibit a wipe opening angle, as seen in Figures $2 b$ and $c$. Inside the pseudo disc, there is a rotationally supported disc that generates a strong toroidal field (Fig. 2 $2 d$ ), because the magnetic field lines are twisted by the rotation motion of the disc. Both the twisted amplified field and disc rotation drive the outflow (Blandford, \& Payne 1982; Uchida. \& Shibata 1985), as shown in Figure 2 $d, e$ and $f$. Near the protostar, the configuration of the magnetic field lines and the structure of the outflows are very complex (Figs. $2 e$ and $f$ ). However, it is clear that the net magnetic vector is parallel to the $z$-direction or the initial direction of the magnetic field at any scale. In addition, the disc normal and the outflow are directed in the $z$-direction.

For this model (model T00), the angular vector is aligned with the magnetic vectors in the initial cloud. The rotation and magnetic field are anisotropic forces and break the spherical symmetry in the collapsing cloud. However, the alignment between the angular momentum and global magnetic field uniquely determines the direction of objects such as the pseudo disc, rotating disc, outflows and magnetic field at any scale. The alignment of the disc normal, outflows and magnetic field evokes a simple or classical picture of star formation.

\subsection{Rotation Axis Perpendicular to Magnetic Field: $\theta_{0}=90^{\circ}$}

The density and velocity distributions on the $x=0, y=0$ and $z=0$ cutting planes for model T90 are shown in Figure 3. In the star formation process, two anisotropic forces (Lorentz and centrifugal forces) produce a disc-like structure. The magnetic field produces a pseudo disc (Galli \& Shu 1993), while the cloud rotation forms a rotationally supported (or Keplerian) disc around a protostar. The magnetic energy is larger than the rotational energy in the star-forming cloud (Crutcher 1999; Caselli et al. 2002), and the magnetic field 
dissipates in the high density region of the collapsing cloud (Nakano et al. 2002). Thus, the rotationally supported disc appears at the small scale after the pseudo disc forms at the large scale.

As shown in Figures $3 b$ and $e$, two nested discs appear in the star-forming cloud. Since the outer disc corresponding to the pseudo disc forms along the magnetic field line, its long axis is perpendicular to the global magnetic field. On the other hand, the inner disc rapidly rotates, as shown in Figures $3 a, d$ and $g$, and forms along the rotation axis. Thus, the long axis of the inner rotating disc is perpendicular to the initial rotation axis and parallel to the global magnetic field direction. For this model, we adopted the initial rotation axis as being perpendicular to the (uniform) global magnetic field (see Figs. $3 b$ and $e$ ). In this case, the magnetic field (or Lorentz force) cannot produce or reduce other components of the angular momentum, because the initial magnetic direction is perfectly perpendicular to the rotation axis. Therefore, the rotation direction maintains its initial direction, as seen in Figure 3 , The cavity-like structure seen in Figure 3 is caused by the generation of a toroidal field.

The left panel of Figure 4 shows a close-up view of Figure $3 h$ and indicates that a weak outflow appears and creates a density cavity near the protostar. Around the protostar, the magnetic field lines are strongly bent and twisted, because the circumstellar disc is rapidly rotating. Thus, a strong toroidal field is generated, and the magnetic pressure gradient force drives the outflow. The cavities or density gaps seen in Figures $3 b, c, e, f$ and $h$ are created by the amplified magnetic field.

The configuration of the magnetic field lines and the structure of the disc for model T90 at $t_{\mathrm{ps}}=509.7 \mathrm{yr}$ are plotted in Figure 5. At the large scale, although the whole structure of the magnetic field lines is slightly distorted, we can confirm an hourglass-like configuration in the magnetic field lines. A similar configuration of the magnetic field lines can be confirmed in observations (e.g. Shinnaga et al. 2012). In Figures $5 b$ and $c$, we can infer that a disc-like structure forms along the magnetic field lines, in which the disc is gradually tilted. Thus, the disc is formed by the magnetic effect at the large scale of $\gtrsim 370 \mathrm{au}$.

At the middle scale (Figs. 5]d and $e$ ), although coherent magnetic fields are realized, their direction differs from that of the large-scale field. Since the magnetic field dissipates and magnetic braking weakens, a rotating disc forms along the initial rotation axis at this scale ( $\lesssim 100 \mathrm{au}$ ). As the rotating disc forms, the weak magnetic fields are passively transformed and are crushed or pushed against the disc long axis or along the disc surface. Then, magnetic field lines along the disc short axis appear, as shown in Figures $5 d$ and $e$. At the small scale, 
a toroidal field is generated along the rotation axis of the circumstellar disc due to the disc rotation (Fig. [5f). This configuration of the magnetic field lines was thoroughly investigated in Machida et al. (2006).

For model T90, the rotation axis is maintained in the $x$-direction, while the direction of the local magnetic field significantly varies at different scales. To determine, or visualize, the directions of the magnetic field and angular momentum at different scales, we averaged the magnetic field and angular momentum within a radius (magnetic field) or in a high density region (angular momentum) at each epoch, according to equations (1)-(6) of Paper I. As described in Paper I, we calculated the magnetic field direction in a spherical volume within a radius $r_{\text {th }}=10,100$ and $100 \mathrm{au}$, and averaged it by the volume (see eq. [6] of Paper I). The angular momentum direction was calculated and averaged by the mass in a region of $n>n_{\mathrm{th}}$, in which threshold densities of $n_{\mathrm{th}}=10^{7}, 10^{10}$ and $10^{13} \mathrm{~cm}^{-3}$ are adopted (see eq. [5] of paper I).

Figure 6 plots a time series of the directions of the magnetic field (left) and angular momentum (right) during $\sim 500 \mathrm{yr}$ after protostar formation. The figure indicates that the directions of the magnetic field at the small scale differ considerably from the initial direction (i.e. the $z$-axis). In addition, the direction of the magnetic field varies with time, while the rotation direction is maintained in the $x$-direction (i.e. initial direction). The disc forms along the magnetic field lines at the large scale, while it forms along the rotation axis at the small scale. Thus, the disc normal is determined by the balance between the Lorentz and centrifugal forces, and hence the direction of the disc normal varies according to the spatial scale. In addition, the disc normal should change with time. In the following subsections, we show the general case for $0^{\circ}<\theta<90^{\circ}$.

\subsection{Rotation Axis Misaligned with Magnetic Field: $\theta_{0}=30^{\circ}$}

Figure 7 shows the density and velocity distributions on the $x=0, y=0$ and $z=0$ planes for model T30. In the figure, we can confirm that the outflow is strongly ejected by the rotating disc. At the large scale, we can see that a filamentary structure is formed from a pseudo disc, in which the pseudo disc is twisted by the rotation motion (Takahashi et al. 2019). As a whole, the structures seen in this model in Fig. 7 are basically the same as for model T00 (Fig. 1). In both models, the outflow, a (twisted) pseudo disc and a rotationally supported disc appear. The most significant difference between models T00 and T30 is the directions of 
the outflows and disc. These directions in the misalignment model T30 significantly change at different spatial scales (Fig. (7), while the directions do not change in the aligned model T00 (Fig. 1). In the misalignment model, the directions seem to be significantly changed, even for different viewing angles.

Figure 8 shows a three-dimensional view of the magnetic field lines, outflows and disc at $t_{\mathrm{ps}}=670.7 \mathrm{yr}$ for model T30. Although the magnetic field lines have an hourglass-like configuration at the large scale (Figs. $8 a$ and $b$ ), they have a very complicated configuration at the small scale (Fig. $8 c-f$ ). We can also confirm that the disc normal direction differs at each scale. In addition, the outflow direction changes dependent on its velocity, as already shown and discussed in Paper I. In Figures $8 c$ and $8 d d$, the low-velocity outflow components are coloured blue and green, while the high-velocity component is coloured yellow. The panels indicate that the high-velocity components are tilted toward the low-velocity component. As seen in Figures $[8 c$ and $8 d d$, the angle between the $z$-axis and the outflow direction is smaller in the low-velocity component than in the high-velocity component (for details, see Paper I). Thus, the different directions of outflows are nested. Recent ALMA observation also confirmed a different direction for the flows at a very early stage of star formation (Matsushita et al. 2019).

Near the protostar, the magnetic field lines are strongly twisted by the rotation motion of the disc (Fig. 8ff). In addition, the outflow directions are considerably different from the initial direction of the magnetic field. Also, the disc normal is different from the initial direction of the angular momentum (i.e., the $x$-direction). Thus, at the middle and small scales $(\lesssim 1000 \mathrm{au}$ ), the magnetic field lines, outflows and disc have no specific information about the initial (or large-scaled) directions of magnetic field and angular momentum (for details, see $\$ 3.5$.

\subsection{Other Misalignment Models}

In this subsection, we briefly comment on the four models with $\theta_{0}=5,10,60$ and $80^{\circ}$ (models T05, T10, T60 and T80). These models have interesting features, some of which seem to reproduce specific observations.

Figure 9 shows the density and velocity distributions on the $y=0$ plane for the models T05, T10, T60 and T80. For model T05 (Figs. 9 $a-c$ ), the initial small angle difference results in a noticeable tilt of the disc normal and outflow directions toward the $z$-axis. 
In Figure $9 b$, we can see a warped pseudo disc. In addition, the angle difference between the initial magnetic field and angular momentum produces a knotty jet-like configuration (Fig. 9c), which is often seen in observations (e.g. Cunningham et al. 2009). The speed of the jet-like flow exceeds $20 \mathrm{~km} \mathrm{~s}^{-1}$.

When the angle difference is as small as $\theta_{0}=10^{\circ}$ (model T10), the disc around the protostar is highly distorted, as shown in Figure $9 d$. In this model, the disc normal and outflow directions rapidly change with time (for details, see $\S 3.5$ ). Therefore, the outflow has a wide opening angle (Fig. [9e and $f$ ). Wide-angle outflows are also seen in some observations (e.g. Velusamy \& Langer 1998).

In model T60, we can confirm a strong mass ejection. In Figure 10i, high-density clumps are embedded in the outflow. The inclined outflow interacts with the pseudo disc and strips its surface. The stripped gas from the pseudo disc is ejected as a part of the outflow. The interaction between the outflow and pseudo disc produces some gas clumps in the outflow, which is also seen in observations (Plunkett et al. 2013; Osorio et al. 2017).

The outflow direction drastically changes over time in model T80. In Figure 9l, we can see a quadrupole-like shock, which is caused by outflows with different directions. This kind of quadrupolar or multipolar outflow is confirmed in observations (Gerin et al. 2015). In this model, the outflow propagates roughly along the $x$-axis at early times. Then, the outflow changes direction and propagates mainly close to the $z$-direction. As a result, a quadrupolar cavity is formed.

Figure 10 plots three-dimensional views of the magnetic field lines, high-density region and outflow for model T80. As seen in Figure $10 \mathrm{~b}$, the ejected knot changes the local configuration of the magnetic field lines. Although the outflow is driven by the magnetic effect, the magnetic field is also affected by the outflow. In Figure 10c, a quadrupolar outflow is indicated by arrows. When the initial rotation axis is highly inclined from the global field, the disc normal and outflow directions rapidly change over time (see, \$3.5).

As shown in Figure 9, the initial angle difference produces various morphologies for the outflows. Thus, the angle difference could explain the different morphologies seen in observations (e.g. Velusamy et al. 2014). 


\subsection{Directions of Magnetic Field, Outflow and Disc}

The structures of the magnetic field, outflow and disc at $t_{\mathrm{ps}} \simeq 500 \mathrm{yr}$ for all the models are summarized in Figure 11, in which the spatial scale and viewing angle are the same in all the panels. In all the models, the initial magnetic field is set to be parallel to the $z$-direction. Thus, the initial direction of the magnetic field is upward in Figure 11. At the scale of $\sim 1000 \mathrm{au}$, we can confirm an hourglass-like configuration of the magnetic field in all the models. However, except for model T00, the net magnetic field directions seem to be inclined from the initial direction (i.e. $z$-direction), especially for models T30, T45, T60, T80, T85 and T90. The net magnetic field directions gradually change from the initial direction as the spatial scale decreases.

The figure also shows that the outflow directions differ in each model and they are not aligned with the initial direction of the magnetic field (i.e. the $z$-direction), except for model T00. The outflow direction is roughly parallel to the $z$-axis for models with small $\theta_{0}$, T00, T05 and T10. On the other hand, the outflow directions are considerably inclined from the initial magnetic field direction for models with large $\theta_{0}$, T30, T45, T60, T80 and T85. In addition, in the models T10, T30, T45, T60 and T80, the outflow directions differ in every velocity component. For example, in model T10, the low-velocity component (blue surface; $v_{r} \simeq 3 \mathrm{~km} \mathrm{~s}^{-1}$ ) is roughly parallel to the $z$-axis, while the high-velocity component (green or orange surface; $v_{r} \sim 35 \mathrm{~km} \mathrm{~s}^{-1}$ ) is substantially inclined from the $z$-axis. Thus, the propagation directions of the outflows differ in every velocity component. Therefore, in addition to the scale of the outflow, we need to pay attention to the outflow velocity to identify the outflow direction.

Next, in Figure 11 we provide an overview of the directions of the disc-like structure (red iso-density surface at the center) and outflow (blue, green and orange iso-velocity surfaces). Note that in Figure 11, the surface density of the disc-like structure is set to be $3.9 \times 10^{8} \mathrm{~cm}^{-3}$. Thus, the disc seen in the figure corresponds to a pseudo disc, not a rotationally supported disc (Machida et al. 2006). The directions of the rotationally supported disc are shown in Figure 13. In Figure 11, the outflow is aligned with the disc normal only for model T00 (Fig. 11 a). In models T10, T30, T45 and T60, the relative angle between the disc normal and the outflow seems to be about $90^{\circ}$. Thus, the outflow direction is roughly perpendicular to the disc normal at the scale of $\sim 1000 \mathrm{au}$. For model T85, the outflow seems to be aligned 
with the disc normal. These results shows that, at this scale, there is no very clear correlation between the magnetic field, outflow and disc normal directions.

To determine the time variation of the directions of the magnetic field, disc (or angular momentum) and outflows, the directions at each time are plotted in Figure 12, Note that, in Paper I, we showed that the disc normal $\boldsymbol{n}$ is almost parallel to the angular momentum $\boldsymbol{J}$ in the high density region $n \gtrsim 10^{10} \mathrm{~cm}^{-3}$, above which the outflow emerges (Machida et al. 2006, 2007; Machida \& Hosokawa 2013). Thus, to avoid complexity, we only plotted the directions of the angular momentum instead of the disc normal. As described above, the derivation of the directions are fully described in Paper I. A good agreement between the disc normal and angular momentum in the high density region was confirmed in Paper I.

The directions of the magnetic field at different scales are plotted in the left column of Figure 12, In each model, the direction of the net magnetic field at a scale of $1000 \mathrm{au}$ roughly maintains its initial direction, i.e. the $z$-direction (see the blue dots in the left column). On the other hand, the net magnetic field at the scale of $100 \mathrm{au}$ (green dots) is significantly inclined from the initial direction. Furthermore, at the scale of 10 au (red dots), the magnetic field direction significantly changes over time, and considerably differs from the initial direction. Thus, the magnetic fields at the small scale of $\lesssim 100-1000$ au do not retain their initial or large-scaled directions.

The directions of the angular momentum are plotted in the middle column of Figure 12 , As described in $\$ 2$, the rotation axis is inclined from the $z$-axis to the $x$-axis by $\theta_{0}$. The rotationally supported disc forms in the region of $z 10^{10} \mathrm{~cm}^{-3}$ where the magnetic field dissipates and magnetic braking becomes ineffective (Machida et al. 2011; Tomida et al. 2015; Tsukamoto et al. 2015). The discs with a high density of $\geqslant 10^{10} \mathrm{~cm}^{-3}$ (green dots) and $\geqslant 10^{13} \mathrm{~cm}^{-3}$ (red dots) seem to keep the initial direction of the angular momentum while staying around the initial direction, which results in oscillation of the disc surface where the protostellar outflow emerges.

The structure of the rotationally supported disc at $t_{\mathrm{ps}} \simeq 500 \mathrm{yr}$ for all models is plotted in Figure 13. The figure indicates that the disc gradually inclines from the $z$-direction as the initial mutual angle $\theta_{0}$ increases. A comparison of Figure 11 and Figure 13 indicates that, in each model, the outflow direction near its driving region is roughly aligned with the disc normal. Note that the viewing angle is the same in Figures 11 and 13. Thus, the outflow emerges along the local disc normal. The disc normal direction differs at every scale and for every density. In the middle column of Figure 12, the direction of the angular momentum (c) 2019 RAS, MNRAS 000, 1\}?? 


\section{$14 \quad$ Machida et al.}

at the low density of $n_{\mathrm{th}}=10^{10} \mathrm{~cm}^{-3}$ is slightly different from that at the high density of $n_{\mathrm{th}}=10^{13} \mathrm{~cm}^{-3}$, which indicates that the disc normal differs at every scale. However, the difference in the angles is not very large. A recent ALMA observation has revealed the misalignment rotation axis or the disc normal (Sakai et al. 2019).

The disc normal direction also changes with time, indicating that the disc normal oscillates. The oscillation of the rotation axis results in different directions of the outflow. In Figure 12, we can confirm that the disc normal direction nearly corresponds to the outflow directions. Roughly, the angular momentum direction of the low-density disc region corresponds to the low-velocity outflow, while that of the high density disc region corresponds to the high-velocity flow, as discussed in Matsushita et al. (2019).

\section{DISCUSSION}

\subsection{Mass of Protostar, Disc and Outflow, and Outflow Momentum}

Many past studies have investigated the star formation process assuming that the initial rotation axis is parallel to the global magnetic field (Tomisaka 2002; Baneriee \& Pudritz 2006; Commercon et al. 2011; Seifried et al. 2012; (Tomida et al. 2013; Machida et al. 2014).

These studies could well reproduce the objects and phenomena observed in star-forming regions, such as pseudo discs, rotationally supported discs and outflows. In this study, we assumed that the rotation angle is inclined toward the global magnetic field. Our simulation could also reproduce pseudo discs, rotationally supported discs and outflows, showing that when the initial rotation axis is not aligned with the global magnetic field, the star formation process does not change qualitatively. On the other hand, our results showed that it is important to pay attention to vector quantities, such as angular momentum and outflow momentum, as their directions change over time. In this section, we compare some physical quantities (or scalar quantities) between the models.

Figure $14 a$ shows the time evolution of the protostellar mass. The protostellar mass is estimated as

$$
M_{\mathrm{ps}}=\int_{n>10^{18} \mathrm{~cm}^{-3} \text { and } v_{r}<1 \mathrm{~km} \mathrm{~s}^{-1}} \rho d v,
$$

where the condition $v_{r}<1 \mathrm{kms}^{-1}$ is imposed so as to exclude the high-density outflow

component (Machida \& Basu 2019). The figure indicates that the initial angle difference does not significantly affect the determination of the protostellar mass. The protostellar mass 
for all models is in the range of $0.01 M_{\odot}<M_{\mathrm{ps}}<0.02 M_{\odot}$ at $t_{\mathrm{ps}}=30 \mathrm{yr}$ and $0.02 M_{\odot}<$ $M_{\mathrm{ps}}<0.04 M_{\odot}$ at $t_{\mathrm{ps}}=500 \mathrm{yr}$. Thus, the mass accretion rate is estimated to be $\sim 4^{-}$ $8 \times 10^{-5} M_{\odot} \mathrm{yr}^{-1}$. The difference in the mass and mass accretion rate among the models is within a factor of two.

Figure $14 b$ plots the disc mass for all models against the elapsed time after protostar formation. The disc mass is estimated according to the procedure described in Paper I. Unlike the protostellar mass, there is over one order of magnitude difference in the disc mass. At $t_{\mathrm{ps}} \sim 500 \mathrm{yr}$, the disc mass for model T80 is $1.6 \times 10^{-3} M_{\odot}$, while that for model T05 is $5.0 \times 10^{-2} M_{\odot}$. The disc mass is smaller in models with lager $\theta_{0}$ than in models with smaller $\theta_{0}$. However, the disc mass for model T90 rapidly increases with time, and roughly catches up with the disc mass of model T00 at $t_{\mathrm{ps}} \sim 500 \mathrm{yr}$. Although further long-term simulations are necessary to determine the disc mass, the diversity of the disc mass may lead to the diversity of planetary systems. A detailed discussion of the angle dependence $\theta_{0}$ on the disc mass and radius is described in Hirano, Machida \& Basu (2019b).

The outflow mass and outflow momentum are plotted in Figure $14 c$ and $d$, respectively. They are estimated as

$$
\begin{aligned}
& M_{\text {out }}=\int_{v_{r}>1 \mathrm{~km} \mathrm{~s}^{-1}} \rho d V, \\
& P_{\text {out }}=\int_{v_{r}>1 \mathrm{~km} \mathrm{~s}^{-1}} \rho v d v,
\end{aligned}
$$

where $v_{r}$ is the radial velocity and $v$ is defined as $v=\left(v_{x}^{2}+v_{y}^{2}+v_{z}^{2}\right)^{1 / 2}$. As shown in $\oiint 3.2$, a very weak outflow appears in model T90. Thus, the outflow mass and momentum in model T90 are significantly smaller than in other models. Furthermore, among the models with $\theta_{0}<90^{\circ}$, the models having larger angles, models T45, T60 and T80, tend to have larger outflow mass and momentum. As shown in Figure 9, in such models, the outflow interacts with the pseudo disc and strips the gas of the pseudo disc. Therefore, such models can have a large outflow mass and momentum. Although there exists a difference in the outflow mass and momentum, the difference between the models is within one order of magnitude at $t_{\mathrm{ps}} \simeq 500 \mathrm{yr}$ except for model T90. Thus, the initial angle difference does not cause a significant difference in the outflow physical quantities. 


\subsection{Disc Direction}

In this study, we adopted a strong magnetic field, as described in \$2, Thus, the angular momentum perpendicular to the magnetic field should be preferentially transferred (Mouschovias \& Paleologou 1979, 1980a .b). Interestingly however, the initial rotation axis seems to be roughly preserved (Fig. 13). In the star-forming cloud, since the gas rapidly collapses in the early phase (or the low-density region), magnetic braking is not fully effective (Machida et al. 2011; Tsukamoto et al. 2018). On the other hand, in the high-density region, the magnetic field dissipates and magnetic braking becomes ineffective. Thus, a rotationally supported disc forms almost along the initial rotation axis (Machida 2014; Hirano \& Machida 2019).

After disc formation, the angular momentum of the disc is transferred by the outflow and magnetic braking. The disc first forms in the magnetically inactive region (Machida et al. 2014, 2016); the magnetic field lines are passively bent roughly along the disc normal direction. With such a magnetic configuration, the magnetic field transfers the angular momentum preferentially perpendicular to the disc normal (Matsumoto \& Tomisaka 2004). As a result, although the disc normal oscillates, it thereby maintains the initial direction during the main accretion stage.

\subsection{Outflow Mass and Velocity}

As described in \$1, there exists a discrepancy in the outflow velocity between simulations and observations. The outflow velocities calculated in many past simulations are within $\sim \mathrm{km} \mathrm{s}^{-1}$ Inutsuka 2012, and references therein), while those in observations are $\sim 10-100 \mathrm{~km} \mathrm{~s}^{-1}$ (Arce et al. 2007). In many simulations, the region around the protostar is masked by sink cells or sink particles. Thus, the high-velocity component never appears in such simulations, because it is driven near the protostellar surface (Machida et al. 2006). On the other hand, since this study resolved the protostar itself, the high velocity component appears.

Figure 15 plots the outflow mass against the outflow radial velocity $v_{\text {rad }}$ at the end of the simulation, in which the outflow mass at every velocity is estimated as

$$
M_{\text {enc }}\left(v_{\text {rad }}\right)=\int_{v_{\text {rad }}}^{v_{\text {rad }}+\delta v} \rho d V,
$$

where $\delta v=0.05 \mathrm{kms}^{-1}$ is adopted. As shown in the figure, although the outflow mass is small, the outflow velocity exceeds $\gg 20 \mathrm{~km} \mathrm{~s}^{-1}$ except for model T90. In addition, except for model T90, the maximum outflow velocity during the simulation exceeds $\sim 50-100 \mathrm{~km} \mathrm{~s}^{-1}$. 
A detailed analysis of the outflow and its velocity is beyond the scope of this study, suffice it to note that the outflow velocities in this simulation are comparable to observations.

\section{SUMMARY}

As described in \$1, the star formation process has been investigated in simple settings, in which the initial rotation axis is assumed to be parallel to the global magnetic field in simulations. In this case, the directions of outflow, disc normal and magnetic field lines at any scale are aligned. On the other hand, Hull et al. (2013) pointed out that the outflow directions are not aligned with global magnetic fields. Thus, past numerical simulations seem not to agree with the observations. Some researchers have pointed out that the outflow direction is not always aligned with the global magnetic field, when the initial rotation axis is inclined from the global magnetic field (Matsumoto \& Tomisaka 2004). However, in the past simulations, since the protostar is not resolved with sufficient spatial resolution, the speed of outflow is $<10 \mathrm{~km} \mathrm{~s}^{-1}$, which is significantly slower than that measured in observations. In our previous study (Paper I), we showed that the directions of outflow, disc normal and magnetic field lines are not aligned in the early star formation stage, during which the outflow speed exceeds $\gtrsim 50-100 \mathrm{~km} \mathrm{~s}^{-1}$ (see also Machida \& Basu 2019). However, in Paper I, we only presented a single model $\left(\theta_{0}=45^{\circ}\right)$. Thus, we do not know whether or not the angle difference is generally maintained.

In this study, we investigated the evolution of clouds having different mutual angles between the initial cloud rotation axis and global magnetic field. The simulations showed that the directions of outflow, disc and local magnetic field are never aligned during the early accretion stage. In summary, there are no clear correlations in angles between the outflow, local magnetic field and disc.

The disc normal is determined by the balance between the centrifugal and Lorentz forces. In star-forming clouds, since the rotation degree and magnetic field strength differ at different spatial scales and in different epochs, the disc normal changes accordingly. In addition, since the protostellar outflow is driven by the rotation and magnetic field around the disc, the outflow directions are not simply determined. Furthermore, the outflow itself changes the configuration of the magnetic field, because the magnetic field lines far from the outflow driving region are bent by the ejected clumps. The configuration of the magnetic field is closely related to the magnetic braking and thus the efficiency of the angular momentum 
transfer, which changes the normal direction of the rotationally supported disc. Thus, many factors are related to determining the directions.

The past star formation simulations assuming alignment between the cloud rotation and magnetic field are not unrealistic. Although we should not compare such simulations with observations, especially when interpreting the directions of outflows and magnetic field, the star formation process constructed from the aligned setting is not wrong. Also, in the misalignment cases, the pseudo disc, rotationally supported disc, and low- and high-velocity outflows appear, as in the aligned cases. In addition, the outflow masses and momentum between the aligned and misalignment cases are not significantly different. The only difference between the aligned and misalignment cases is the directions of the outflow, disc and magnetic field lines. The different directions somewhat change the star forming environment at a scale of $\lesssim 1000 \mathrm{au}$, and give rise to complex structures, as seen in observations. At present, we can fairly compare simulations and observations only in the very early phase of star formation. Since the probability of the existence of such early-phase objects is low, we require further long-term calculations to compare star formation between simulations and observations for all phases.

\section{ACKNOWLEDGEMENTS}

The authors would like to thank B. Riaz, K. Tomida and S. Takasao for their helpful contributions. We also thank the referee R. Banerjee for careful reading and useful comment on this paper. The present research used the computational resources of the HPCI system provided by Cyber Science center, Tohoku University and Cybermedia Center, Osaka University through the HPCI System Research Project (Project ID: hp170047, hp180001,

hp190035). The simulations reported in this paper were also performed by the 2018 and 2019 Koubo Kadai on Earth Simulator (NEC SX-ACE) at JAMSTEC. This work was supported by a JSPS Research Fellowship for SH and JSPS KAKENHI Grant Numbers 18J01296 for SH and by 17K05387, 17H06360, and 17H02869, 17KK0096 for MNM.

\section{REFERENCES}

Arce, H. G., Shepherd, D., Gueth, F., et al. 2007, Protostars and Planets V, 245

Banerjee, R., \& Pudritz, R. E. 2006, ApJ, 641, 949

Bate, M. R. 1998, ApJl, 508, L95 
Bate, M. R., Tricco, T. S., \& Price, D. J. 2014, MNRAS, 437, 77

Blandford, R. D., \& Payne, D. G. 1982, MNRAS, 199, 883

Caselli, P., Benson, P. J., Myers, P. C., et al. 2002, ApJ, 572, 238

Larson, R. B., 1969, MNRAS, 145, 271.

Lee, C.-F., Kwon, W., Jhan, K.-S., et al. 2019, ApJ, 879, 101

Commerçon, B., Hennebelle, P., \& Henning, T. 2011, ApJl, 742, L9

Crutcher, R. M. 1999, ApJ, 520, 706

Cunningham, N. J., Moeckel, N., \& Bally, J. 2009, ApJ, 692, 943

Gerin, M., Pety, J., Fuente, A., et al. 2015, A\&A, 577, L2

Galli, D., \& Shu, F. H. 1993, ApJ, 417, 220

Girart, J. M., Rao, R., \& Marrone, D. P. 2006, Science, 313, 812

Girart, J. M., Beltrán, M. T., Zhang, Q., Rao, R., \& Estalella, R. 2009, Science, 324, 1408

Hayashi, C., Nakazawa, K., \& Nakagawa, Y. 1985, Protostars and Planets II, 1100

Hennebelle, P., \& Ciardi, A. 2009, A\&A, 506, L29

Hirano, S., \& Machida, M. N. 2019, MNRAS, 485, 4667 (Paper I)

Hirano, S., \& Machida, M. N. \& Basu, S. 2019 submittted in ApJ.

Hull, C. L. H., Plambeck, R. L., Bolatto, A. D., et al. 2013, ApJ, 768, 159

Inutsuka, S.-i. 2012, Progress of Theoretical and Experimental Physics, 2012, 01A307

Joos, M., Hennebelle, P., \& Ciardi, A. 2012, A\&A, 543, A128

Larson, R. B. 2003, Reports on Progress in Physics, 66, 1651

Machida, M. N., Tomisaka, K., \& Matsumoto, T. 2004, MNRAS, 348, L1

Machida, M. N., Matsumoto, T., Tomisaka, K., \& Hanawa, T. 2005, MNRAS, 362, 369

Machida, M. N., Matsumoto, T., Hanawa, T., et al. 2005b, MNRAS, 362, 382

Machida, M. N., Inutsuka, S.-. ichiro ., \& Matsumoto, T. 2006, ApJL, 647, L151

Machida, M. N., Matsumoto, T., Hanawa, T., \& Tomisaka, K. 2006, ApJ, 645, 1227

Machida, M. N., Inutsuka, S.-i., \& Matsumoto, T. 2007, ApJ, 670, 1198

Machida, M. N., Inutsuka, S.-I., \& Matsumoto, T. 2011, PASJ, 63, 555

Machida, M. N., \& Hosokawa, T. 2013, MNRAS, 431, 1719

Machida, M. N., Inutsuka, S.-i., \& Matsumoto, T. 2014, MNRAS, 438, 2278

Machida, M. N. 2014, ApJL, 796, L17

Machida, M. N., \& Nakamura, T. 2015, MNRAS, 448, 1405

Machida, M. N., Matsumoto, T., \& Inutsuka, S.-i. 2016, MNRAS, 463, 4246

Machida, M. N., Higuchi, K., \& Okuzumi, S. 2018, MNRAS, 473, 3080 
Machida, M. N., \& Basu, S. 2019, ApJ, 876, 149

Masson, J., Chabrier, G., Hennebelle, P., Vaytet, N., \& Commerçon, B. 2016, A\&A, 587, A32

Masunaga, H., \& Inutsuka, S., 2000, ApJ, 531, 350

Matsumoto, T., \& Tomisaka, K. 2004, ApJ, 616, 266

Matsushita, Y., Takahashi, S., Machida, M. N., \& Tomisaka, K. 2019, ApJ, 871, 221

Mouschovias, T. C., \& Paleologou, E. V. 1979, ApJ, 230, 204

Mouschovias, T. C., \& Paleologou, E. V. 1980a, Moon and Planets, 22, 31

Mouschovias, T. C., \& Paleologou, E. V. 1980b, ApJ, 237, 877

Nakano, T., Nishi, R., \& Umebayashi, T. 2002, ApJ, 573, 199

Nakano, T., Hasegawa, T., \& Norman, C. 1995, ApJ, 450, 183

Osorio, M., Díaz-Rodríguez, A. K., Anglada, G., et al. 2017, ApJ, 840, 36

Plunkett, A. L., Arce, H. G., Corder, S. A., et al. 2013, ApJ, 774, 22

Riaz, B., Machida, M. N., \& Stamatellos, D. 2019, MNRAS, 486, 4114

Sakai, N., Hanawa, T., Zhang, Y., et al. 2019, Nature, 565, 206

Seifried, D., Pudritz, R. E., Banerjee, R., et al. 2012, MNRAS, 422, 347

Shinnaga, H., Novak, G., Vaillancourt, J. E., et al. 2012, ApJl, 750, L29

Takahashi, S., Machida, M. N., Tomisaka, K., et al. 2019, ApJ, 872, 70

Tomida, K., Tomisaka, K., Matsumoto, T., et al. 2013, ApJ, 763, 6

Tomida, K., Okuzumi, S., \& Machida, M. N. 2015, ApJ, 801, 117

Tomisaka, K. 2002, ApJ, 575, 306

Tsukamoto, Y., Iwasaki, K., Okuzumi, S., Machida, M. N., \& Inutsuka, S. 2015, MNRAS, 452,278

Tsukamoto, Y., Okuzumi, S., Iwasaki, K., Machida, M. N., \& Inutsuka, S. 2018, ApJ, 868, 22

Uchida, Y., \& Shibata, K. 1985, PASJ, 37, 515

Vaytet, N., Commerçon, B., Masson, J., et al. 2018, A\&A, 615, A5

Velusamy, T., \& Langer, W. D. 1998, Nature, 392, 685

Velusamy, T., Langer, W. D., \& Thompson, T. 2014, ApJ, 783, 6

Wurster, J., \& Bate, M. R. 2019, MNRAS, 486, 2587 


\begin{tabular}{|c|c|c|c|c|c|c|c|c|}
\hline Model & $\theta_{0}$ & $B_{0}[\mathrm{G}]$ & $\Omega_{0}\left[\mathrm{~s}^{-1}\right]$ & $\alpha_{0}$ & $\beta_{0}$ & $\mu_{0}$ & $t_{\mathrm{ps}, \text { end }}[\mathrm{yr}]$ & $M_{\mathrm{ps}, \text { end }}\left[M_{\odot}\right]$ \\
\hline T00 & $0^{\circ}$ & & & & & & 674 & 0.034 \\
\hline T05 & $5^{\circ}$ & & & & & & 418 & 0.021 \\
\hline $\mathrm{T} 10$ & $10^{\circ}$ & & & & & & 786 & 0.045 \\
\hline T30 & $30^{\circ}$ & & & & & & 671 & 0.043 \\
\hline $\mathrm{T} 45$ & $45^{\circ}$ & $5.7 \times 10^{-5}$ & $1.4 \times 10^{-13}$ & 0.39 & 0.026 & 1.2 & 573 & 0.039 \\
\hline T60 & $60^{\circ}$ & & & & & & 573 & 0.033 \\
\hline $\mathrm{T} 80$ & $80^{\circ}$ & & & & & & 682 & 0.025 \\
\hline T85 & $85^{\circ}$ & & & & & & 561 & 0.040 \\
\hline T90 & $90^{\circ}$ & & & & & & 510 & 0.032 \\
\hline
\end{tabular}

Table 1. Model name, initial cloud parameters and calculation results. Column 1 gives the model name. Column 2 gives the parameter $\theta_{0}$. Columns 3 and 4 give the magnetic field strength $B_{0}$ and angular velocity $\Omega_{0}$ at the initial state. Columns 5 and 6 give the ratios of the thermal $\alpha_{0}$ and rotational $\beta_{0}$ energies to the gravitational energy of the initial cloud. Column 7 gives the initial mass-to-flux ratio $\mu_{0}$ normalized by the critical value. Columns 5 and 6 give the elapsed time after protostar formation $t_{\mathrm{ps}, \text { end }}$ and protostellar mass $M_{\mathrm{ps}, \text { end }}$ at the end of each simulation. 

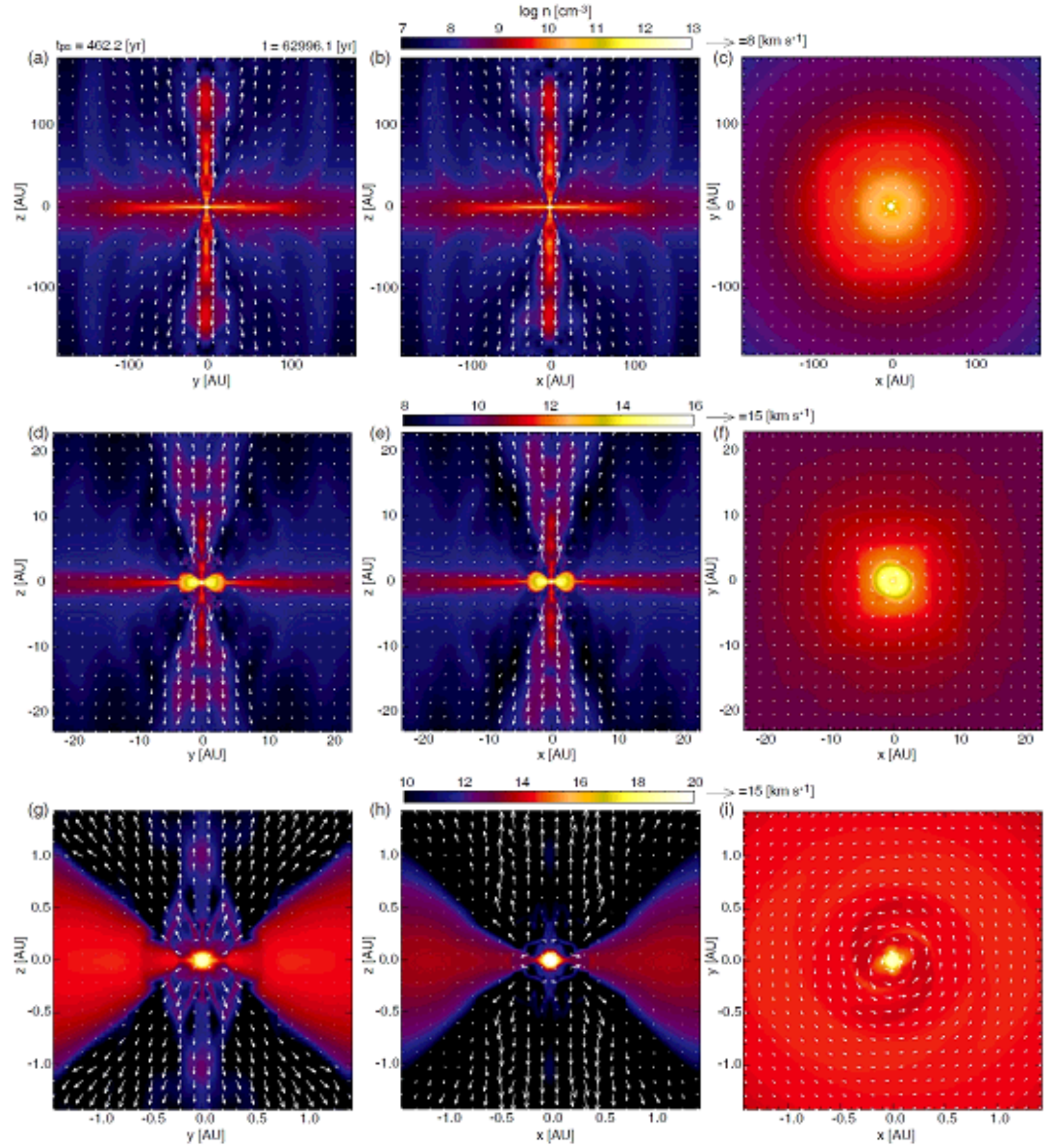

Figure 1. Density (colour) and velocity (arrows) distributions on the $x=0$ (left column), $y=0$ (middle column) and $z=0$ (right column) planes for model T00 with a box size of $295 \mathrm{au}$ (top panels), $37 \mathrm{au}$ (middle panels) and $2.3 \mathrm{au}$ (bottom panels). The elapsed time after protostar formation $t_{\mathrm{ps}}$ and that after the beginning of the cloud collapse $t$ are described in the upper part of panel $(a)$. 


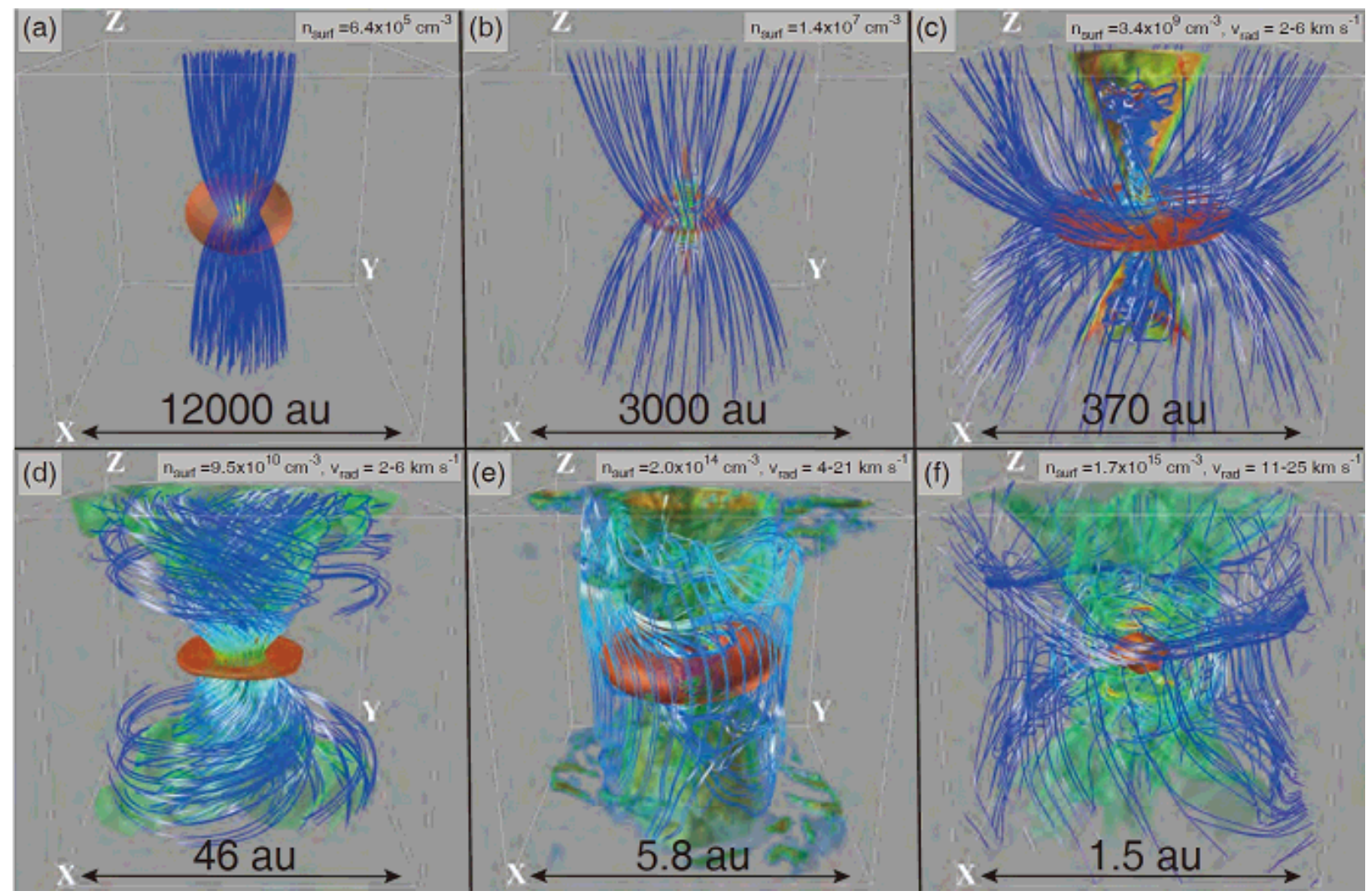

Figure 2. Three-dimensional view of magnetic field lines (blue lines), high-density region (orange iso-density surface) and outflows (blue, green and yellow iso-velocity surfaces) at $t_{\mathrm{ps}}=462.2 \mathrm{yr}$ and $t=62996.1 \mathrm{yr}$ with different spatial scales for model T00. The box size is described in each panel. The density of the iso-density surface $n_{\text {surf }}$ and the velocity range of the outflow $v_{\text {rad }}$ are also described in each panel. The viewing angle is the same in all panels. 
24 Machida et al.
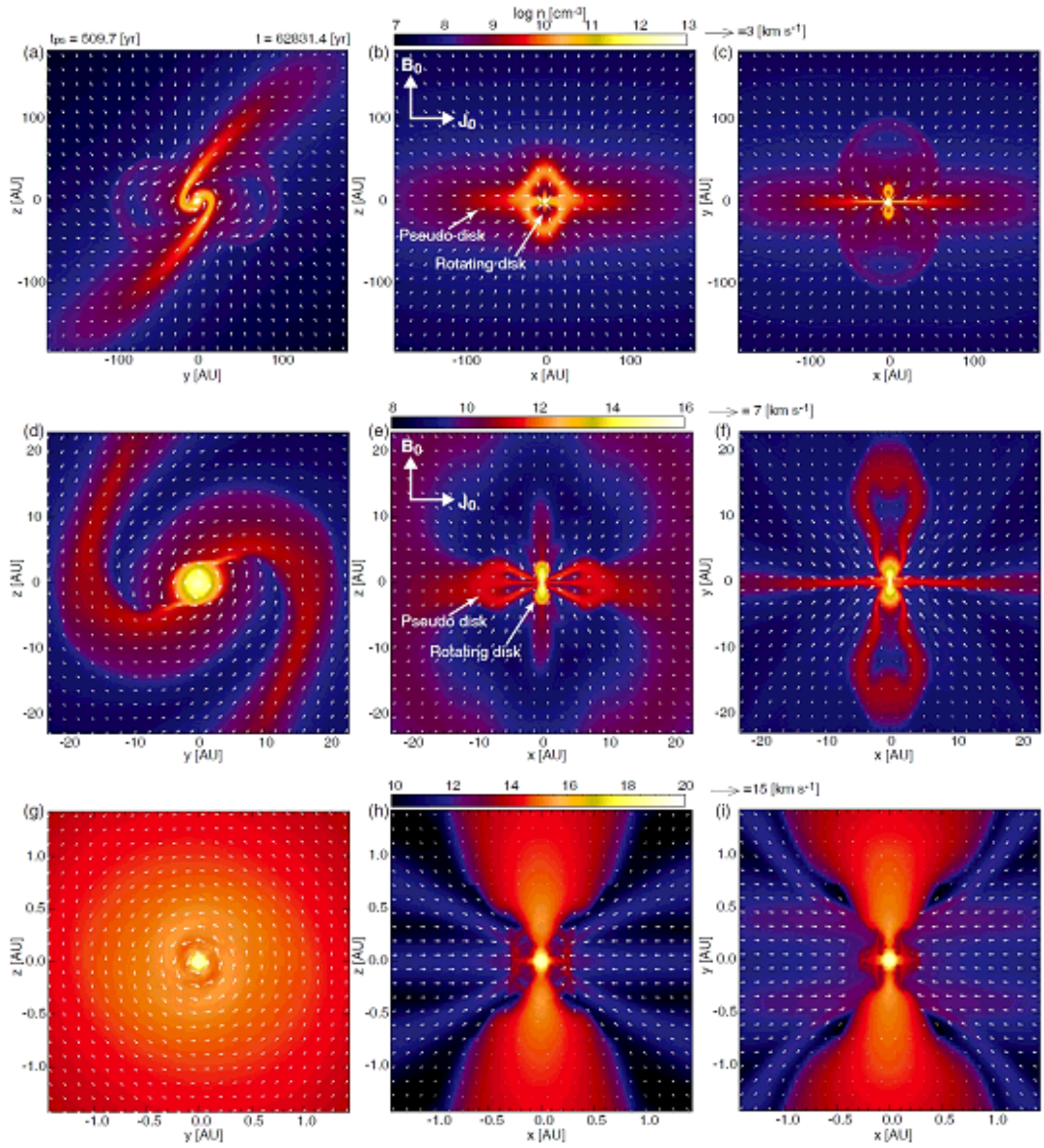

Figure 3. As for Figure 1 but for model T90. The initial direction of the magnetic field and rotation vector are plotted in panels $(b)$ and $(e)$. The pseudo disc and rotating disc are labeled in panels $(b)$ and $(e)$. 


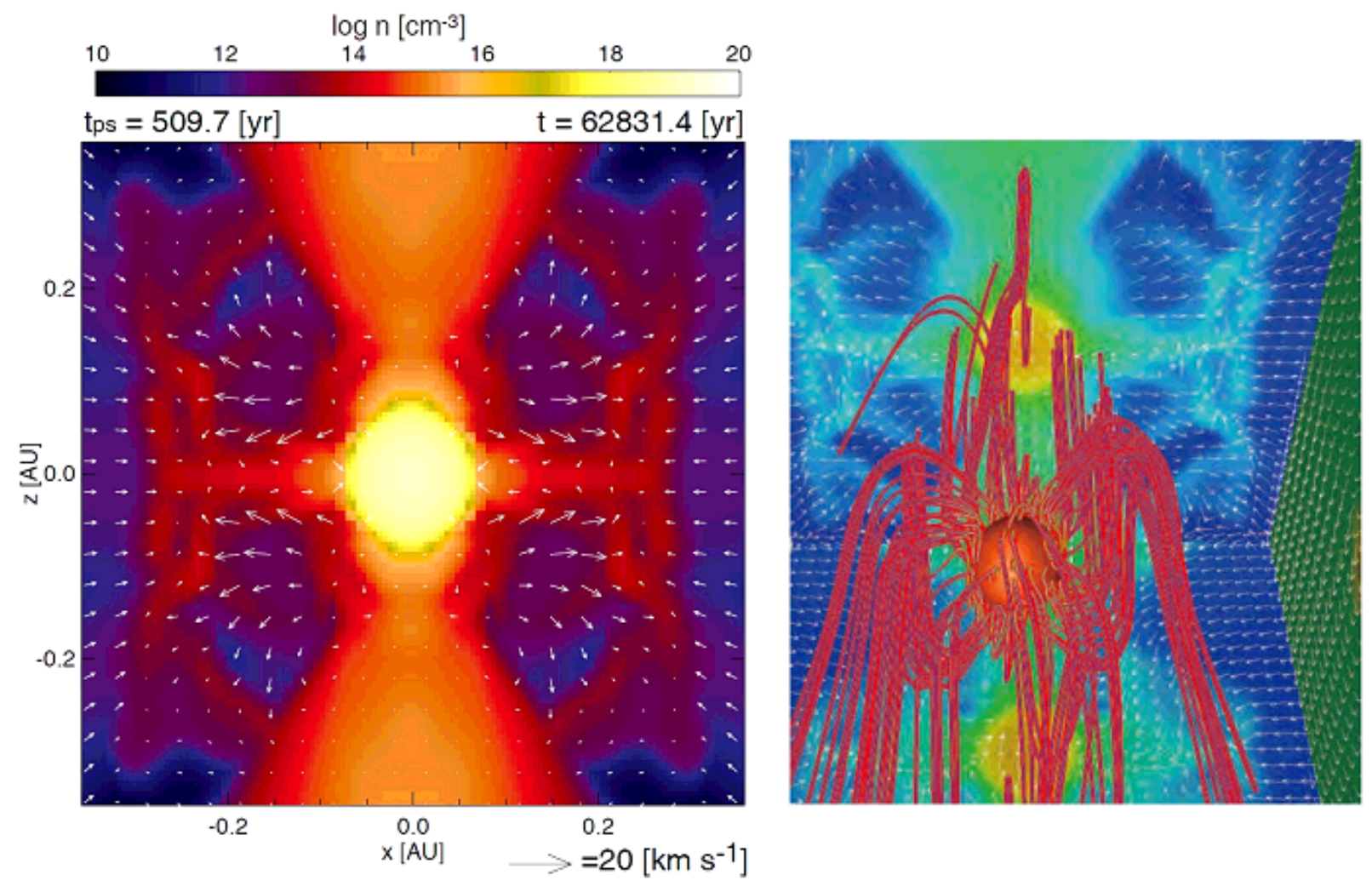

Figure 4. Left: Density (colour) and velocity (arrows) distributions on the $y=0$ plane for model T90. The elapsed time after protostar formation $t_{\mathrm{ps}}$ and that after the beginning of the cloud collapse $t$ are described in the upper part. Right: Threedimensional view of magnetic field lines (red lines) and protostar (orange surface) at the same epoch as in the left panel. The density and velocity distributions on the $x=0, y=0$ and $z=0$ plane are projected on each wall surface. 


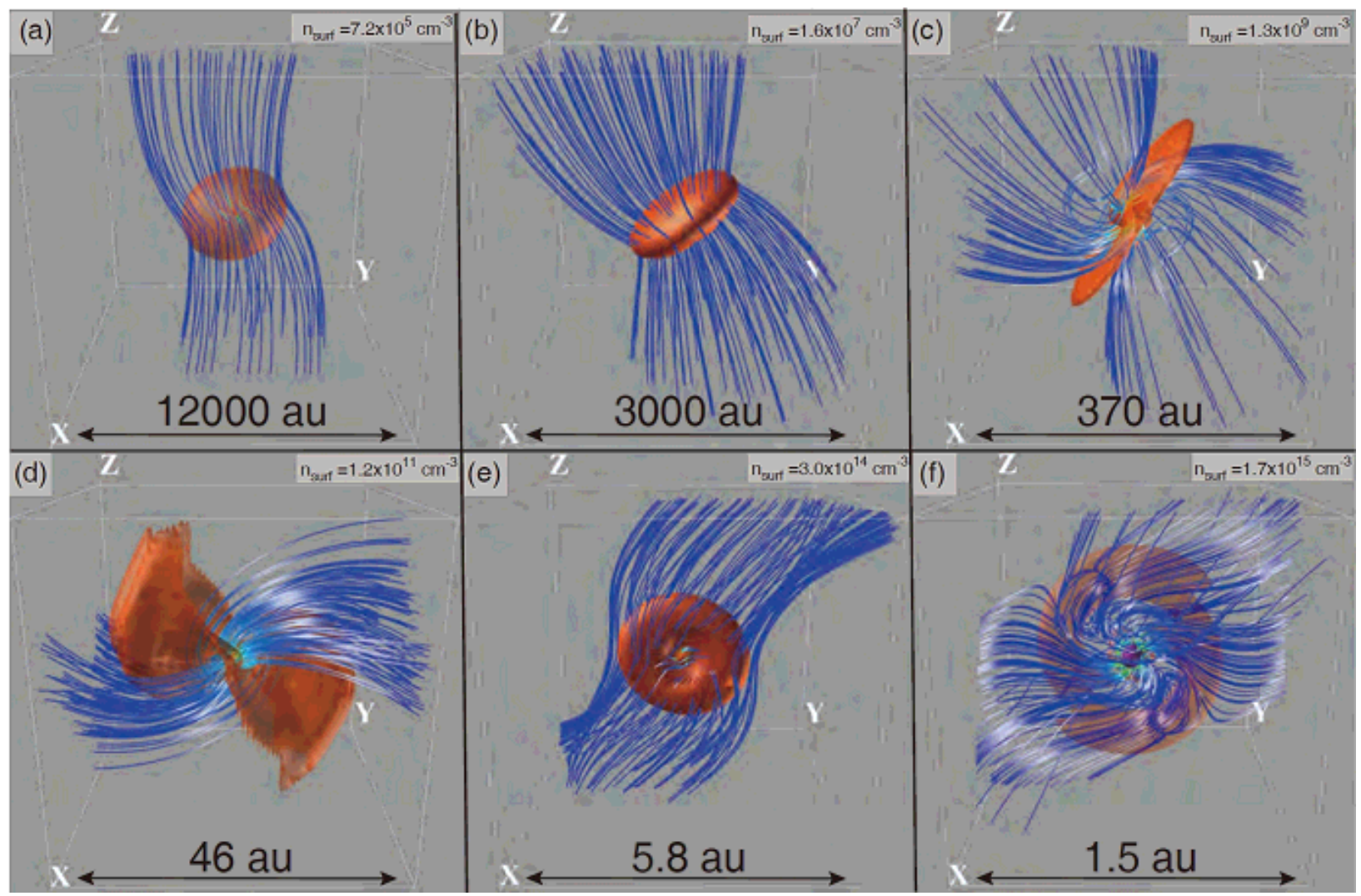

Figure 5. As for Figure 2 but for model T90. 

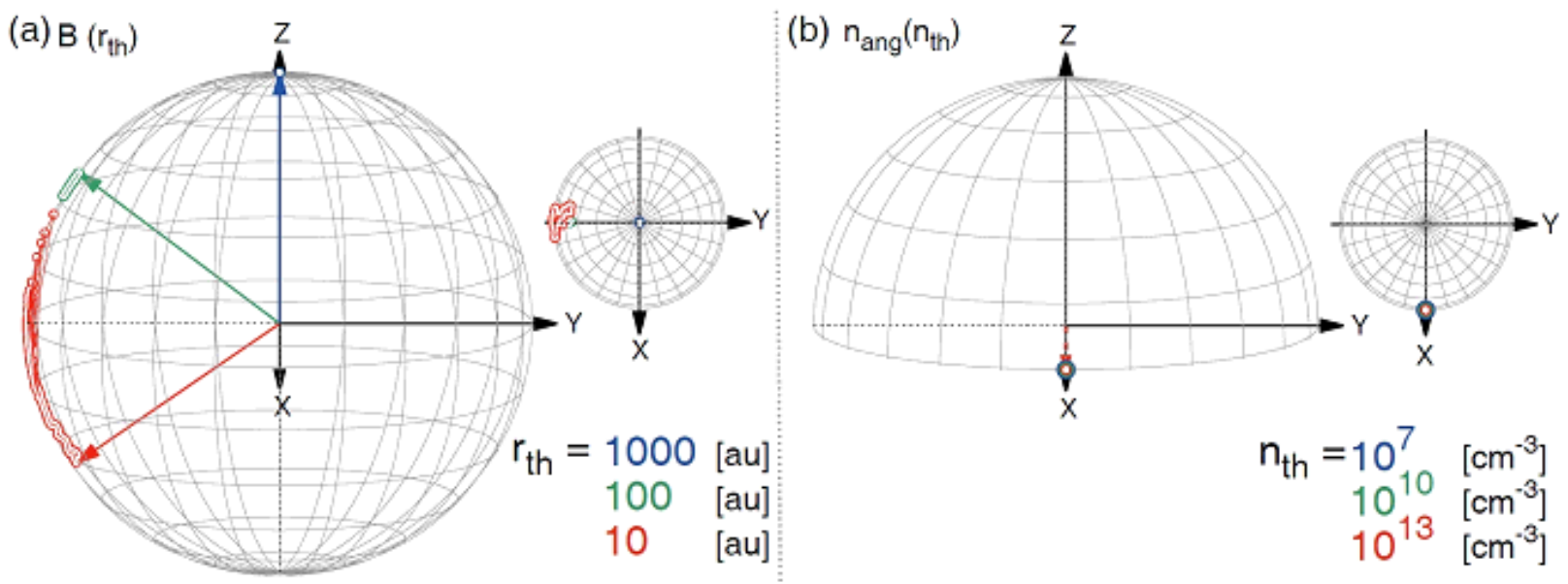

Figure 6. Time evolution of the directions of the magnetic field plotted on a spherical surface (a) and angular momentum plotted on the surface of a hemisphere (b). The magnetic field directions are measured at three different scales of 1000,100 and $10 \mathrm{au}$. The angular vectors are measured for three different threshold densities of $n_{\mathrm{th}}=10^{7}, 10^{10}$ and $10^{13} \mathrm{~cm}^{-3}$. The arrows indicate the direction at the end of the simulation $\left(t_{\mathrm{ps}}=510 \mathrm{yr}\right)$. 
28 Machida et al.
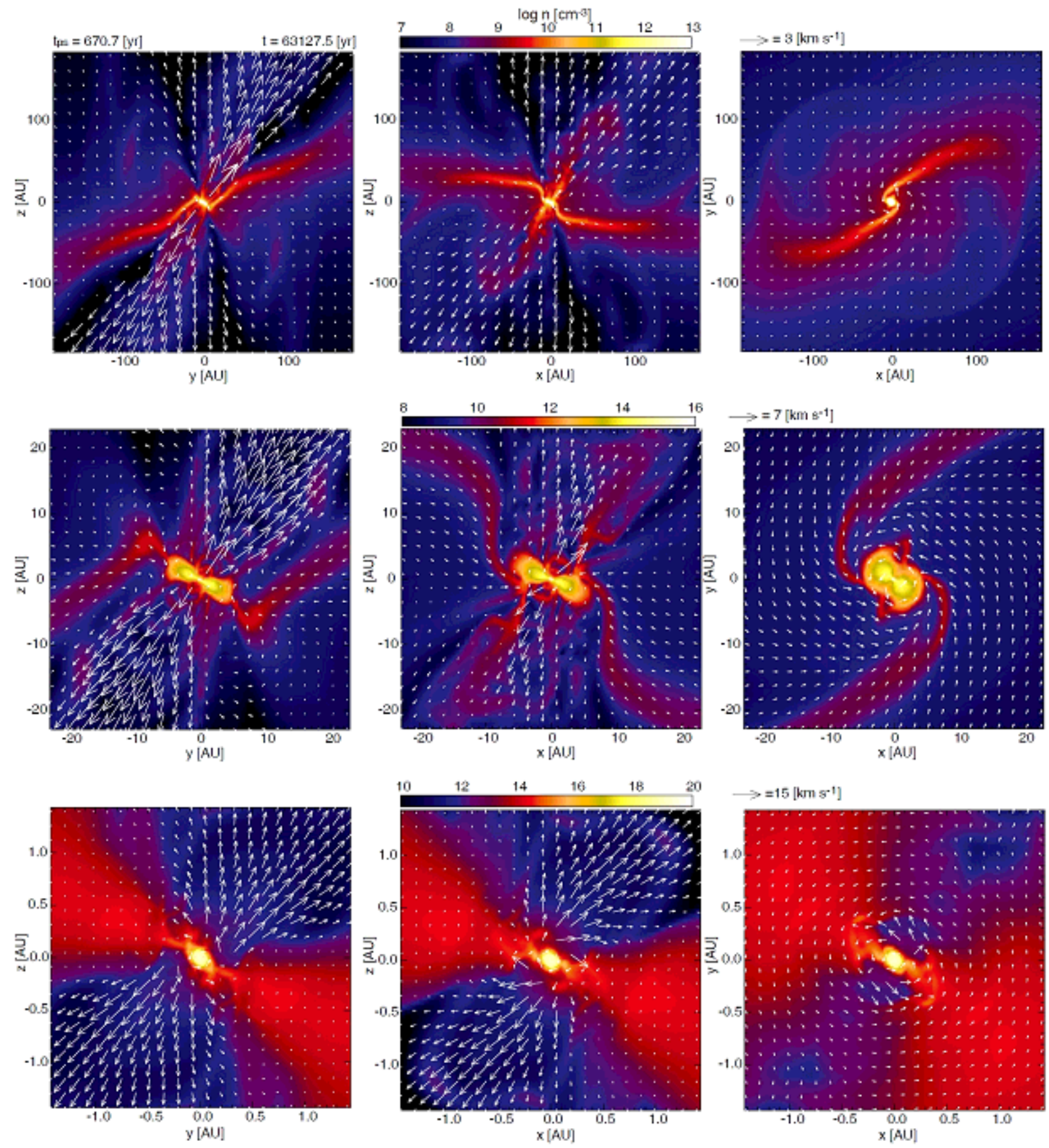

Figure 7. As for Fig. 1, but for model T30. 


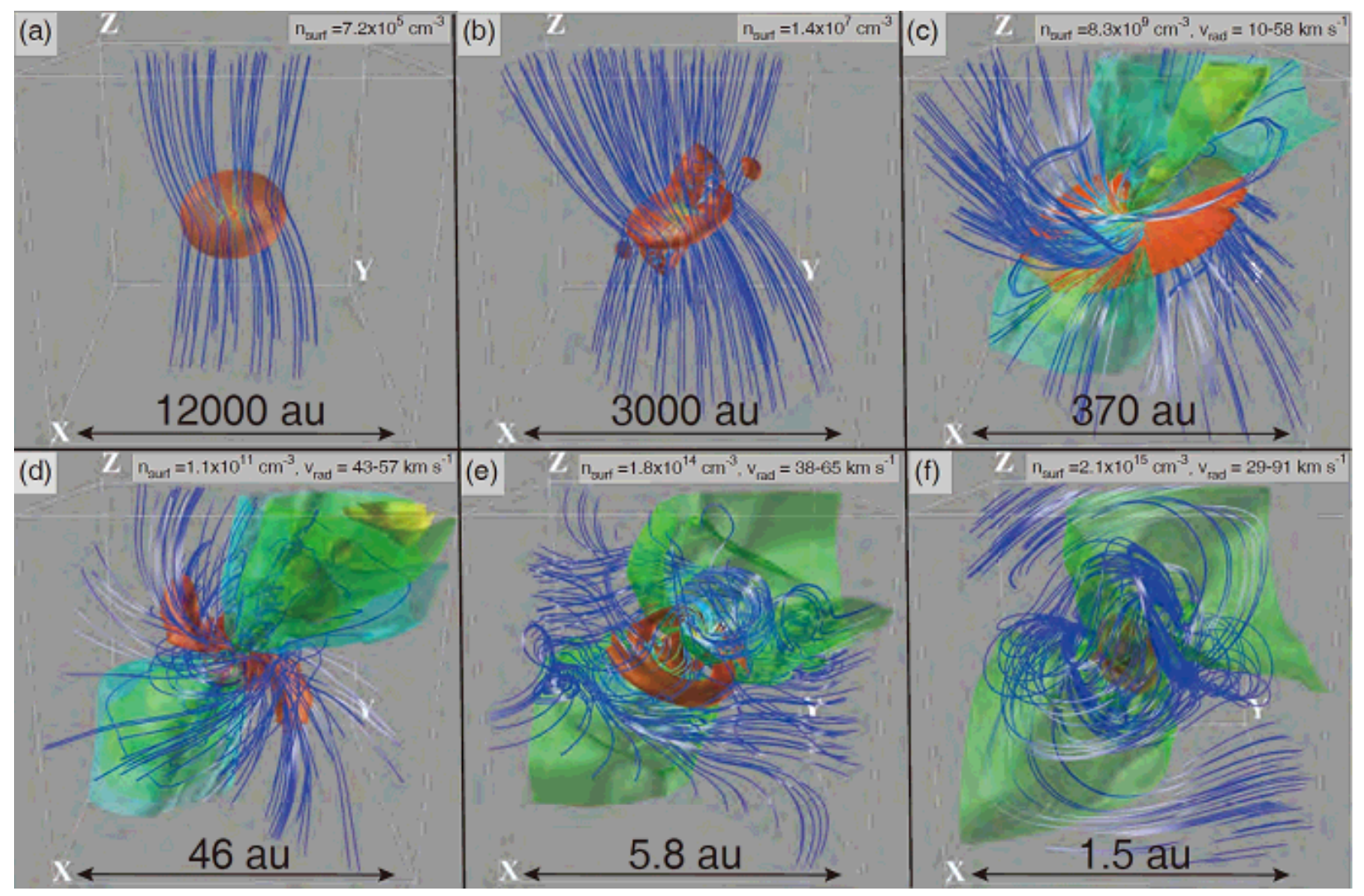

Figure 8. As for Fig. 2, but for model T30. 

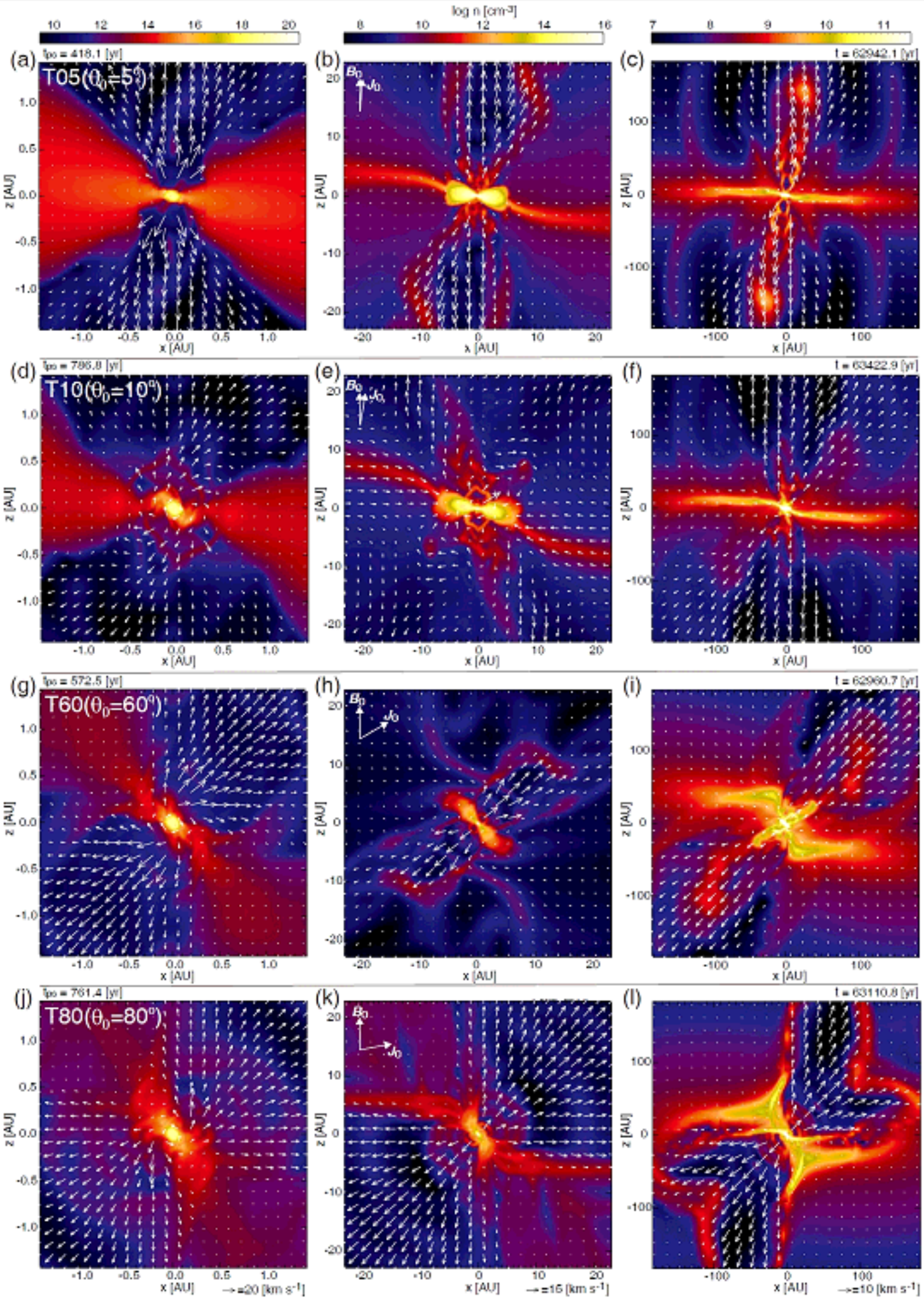

Figure 9. Density (colour) and velocity (arrows) distributions on the $y=0$ plane with a box size of $2.3 \mathrm{au}$ (left), $37 \mathrm{au}$ (middle) and $295 \mathrm{au}$ (right) for models T05, T10, T60 and T80. The elapsed time after protostar formation $t_{\mathrm{ps}}$ and that after the beginning of the cloud collapse $t$ are described in the upper part of each panel. The model name and parameter $\theta_{0}$ are noted in each row. The initial direction of the magnetic field $\boldsymbol{B}_{\mathbf{0}}$ and angular momentum $\boldsymbol{J}_{\mathbf{0}}$ are represented by arrows in panels $(b)$, $(e),(h)$ and $(k)$. 


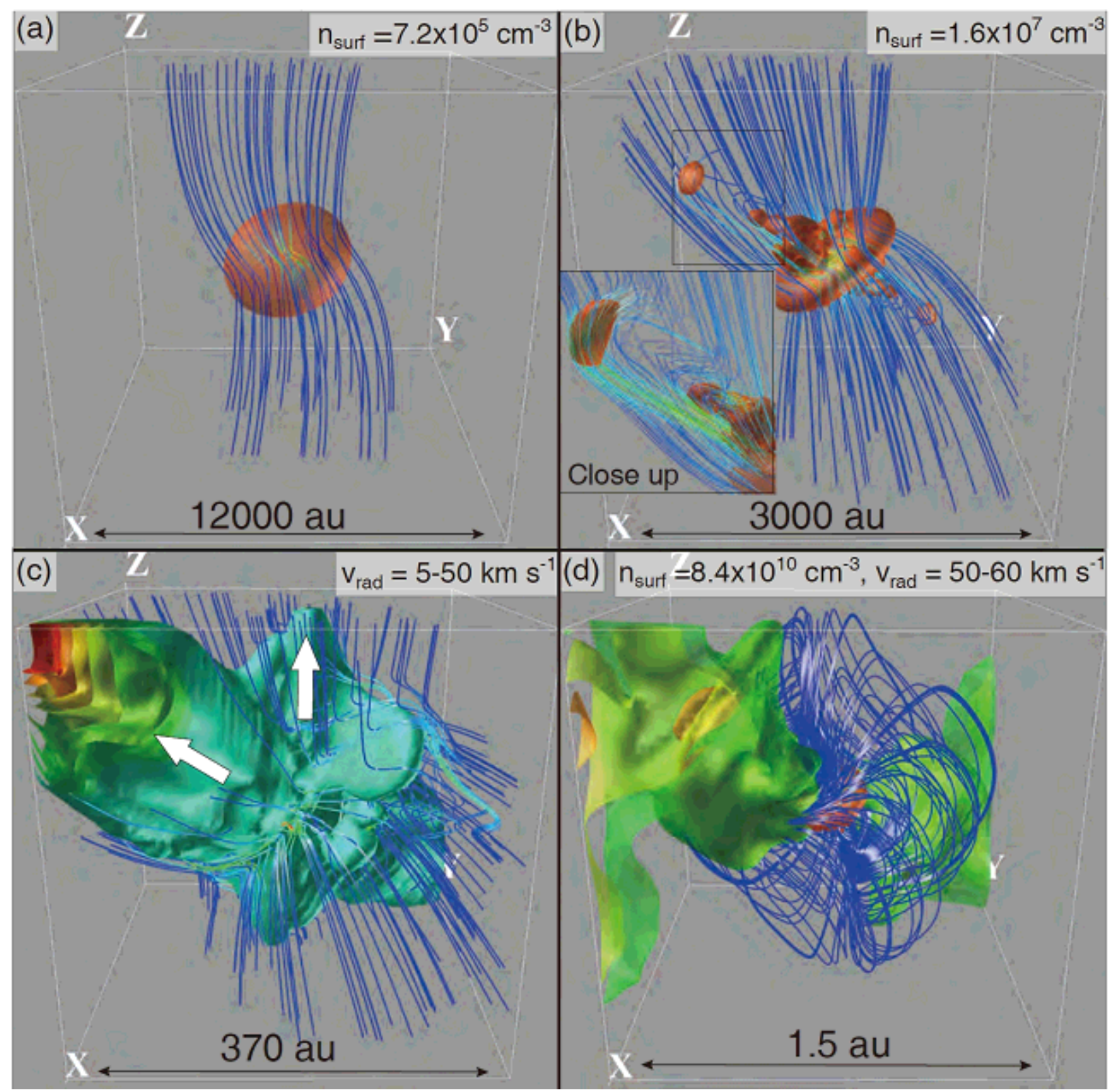

Figure 10. Three-dimensional views of magnetic field lines (blue lines), high-density region (orange iso-density surface around the center) and outflows (blue, green and yellow iso-velocity surfaces) at $t_{\mathrm{ps}}=418.1 \mathrm{yr}$ and $t=62942.1 \mathrm{yr}$ with different spatial scales for model T80. The box size is denoted in each panel. The density of the iso-density surface and the velocity range of the outflow are also described in each panel. A close-up view around a knot is inserted in panel $(b)$. The outflow directions are denoted by arrows in panel $(c)$. 


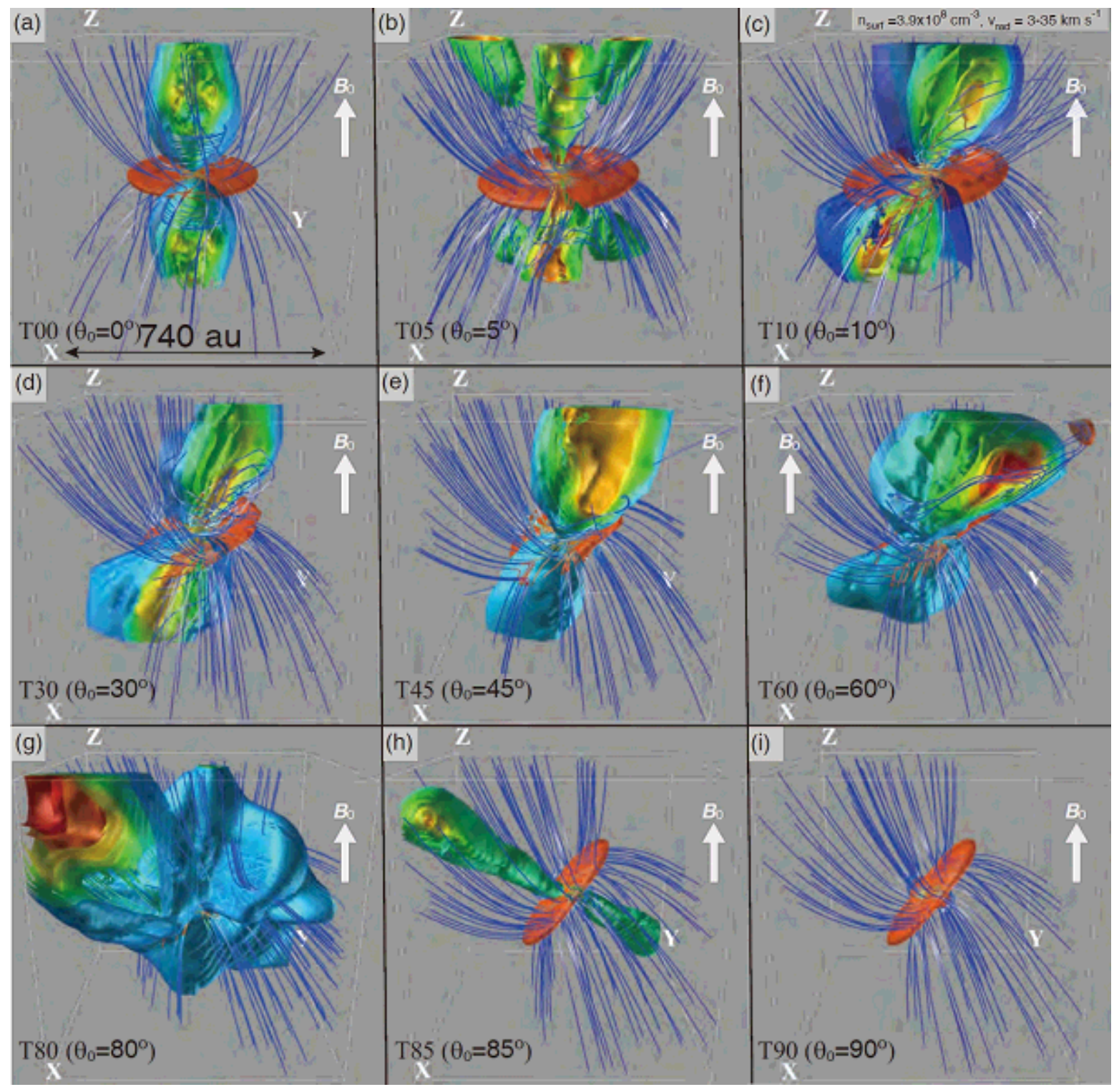

Figure 11. Three-dimensional view of magnetic field lines (blue lines), disc or high-density region (orange iso-density surface at the center) and outflow (green and yellow surfaces) for all models. The box scale is described in panel (a). The surface density of the iso-density surface $n_{\text {surf }}$ and velocity range of the outflow $v_{\text {rad }}$ are described in panel (c). The box scale, surface density and velocity range are the same in all the panels. The initial direction of the magnetic field $\boldsymbol{B}_{0}$ is denoted by an arrow in each panel, in which the initial directions of the magnetic field are also the same among the models. 

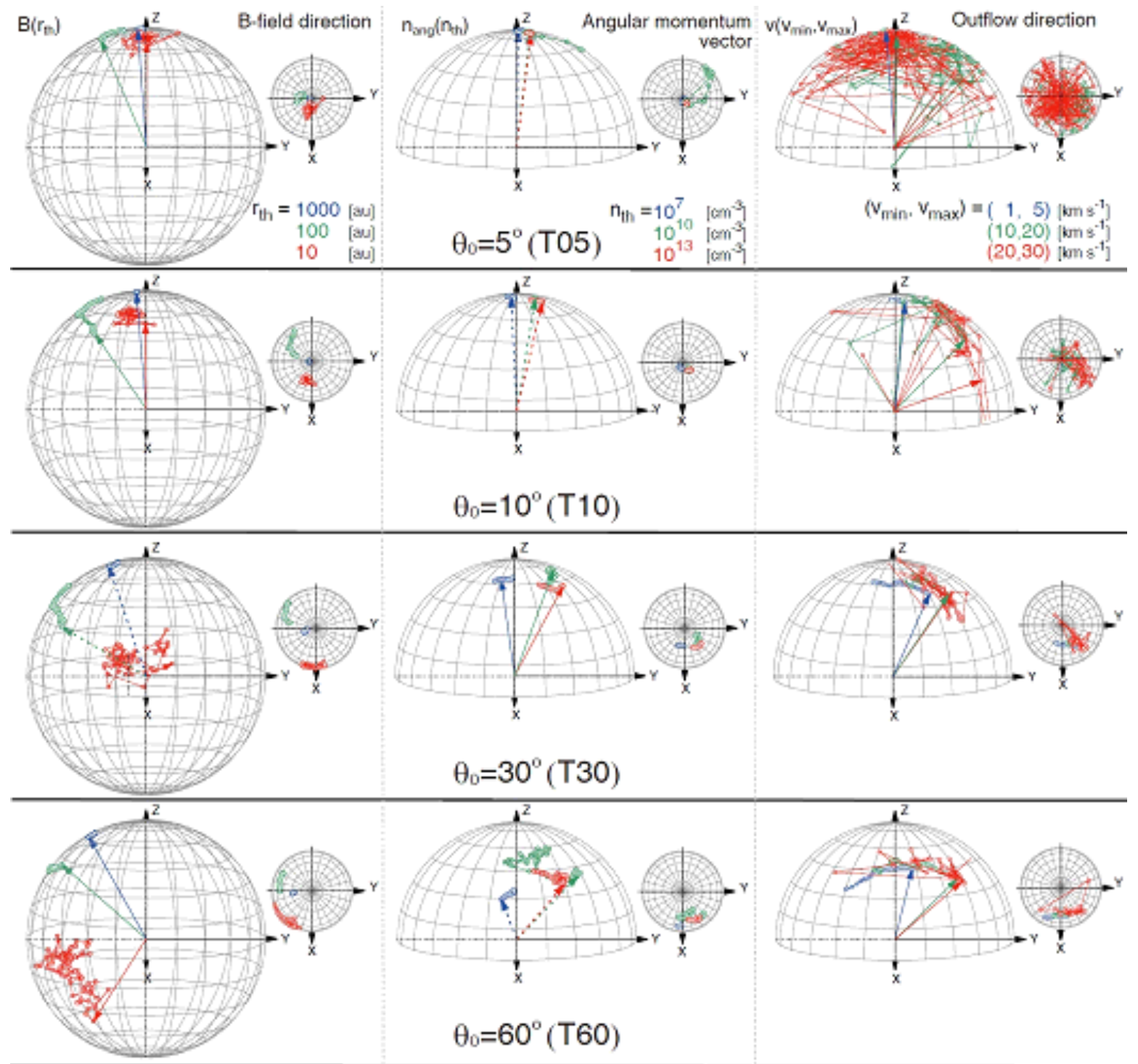

$\theta_{0}=30^{\circ}(\mathrm{T} 30)$
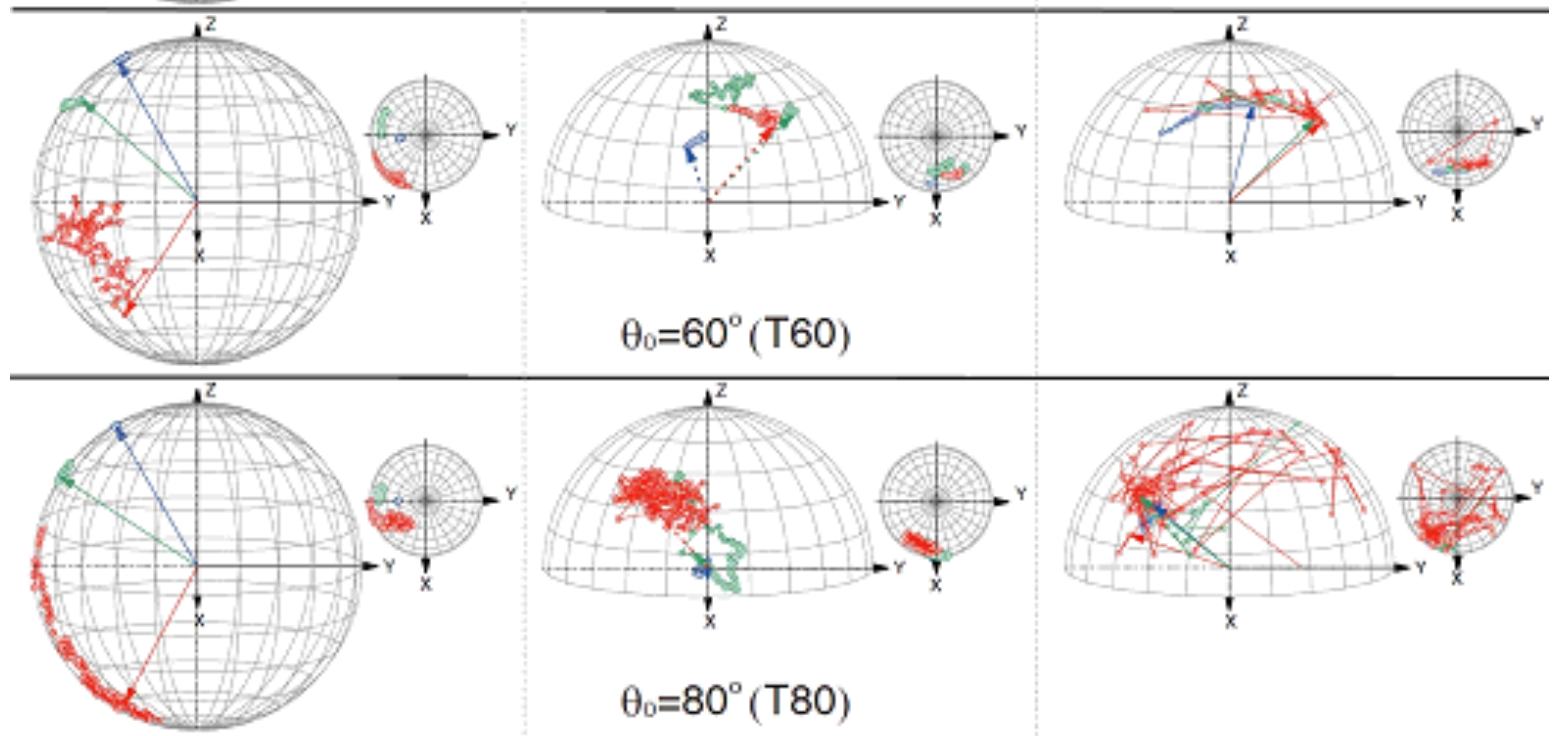

$\theta_{0}=60^{\circ}(\mathrm{T} 60)$
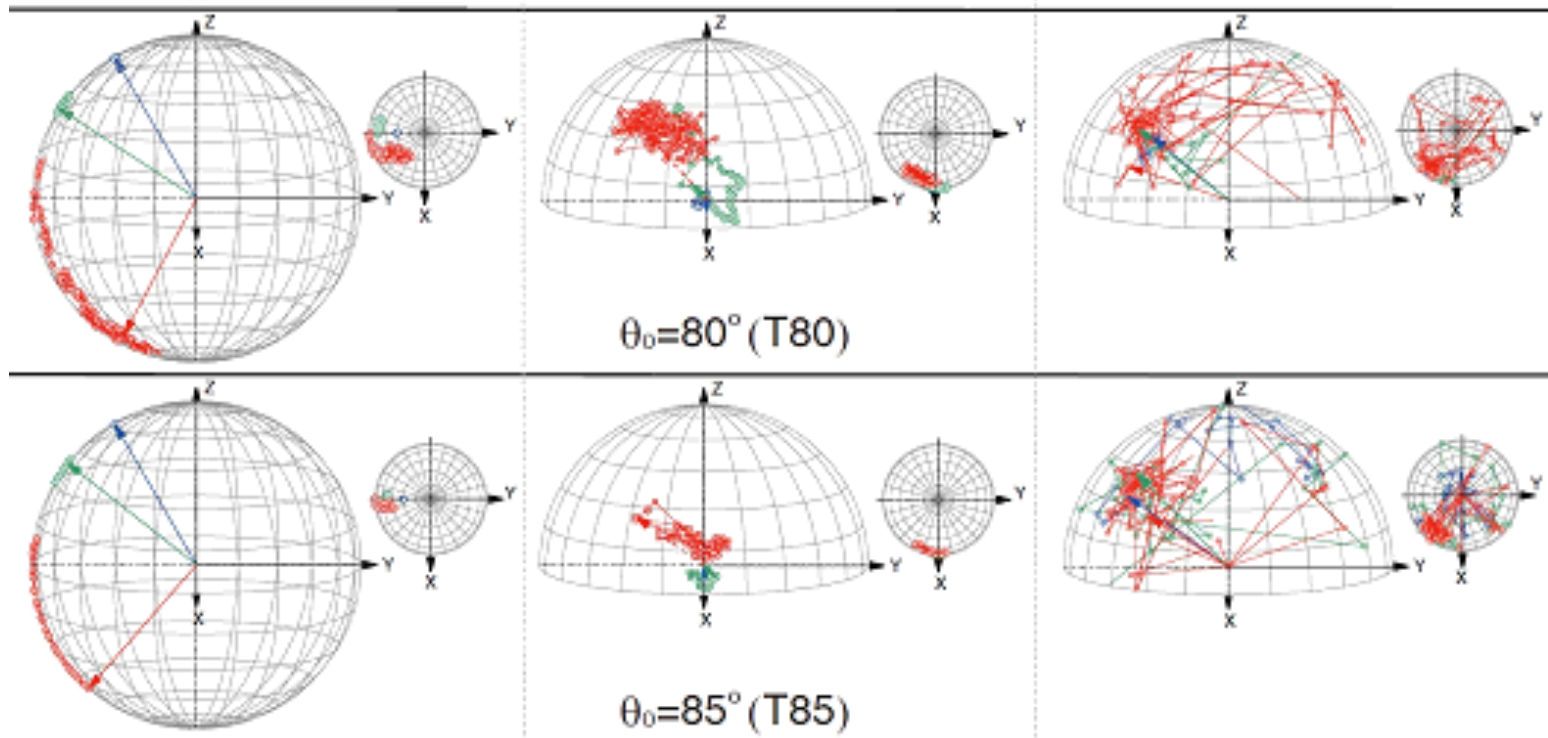

Figure 12. Time evolution of the directions of the magnetic field (left) angular momentum (middle) and outflow (right) plotted on the surface of a sphere and hemisphere for models T05, T10, T30, T60, T80 and T85. The magnetic field directions are measured at three different scales of 1000, 100 and $10 \mathrm{au}$. The angular momentum vectors are measured for three different threshold densities of $n_{\mathrm{th}}=10^{7}, 10^{10}$ and $10^{13} \mathrm{~cm}^{-3}$. The outflows are measured for three different velocity ranges of $\left(v_{\text {min }}\right.$, $\left.v_{\max }\right)=(1,5),(10,20)$ and $(20,30) \mathrm{km} \mathrm{s}^{-1}$. The arrows indicate the direction at the end of the simulation.
(C) 2019 RAS, MNRAS 000, 1 ?? 


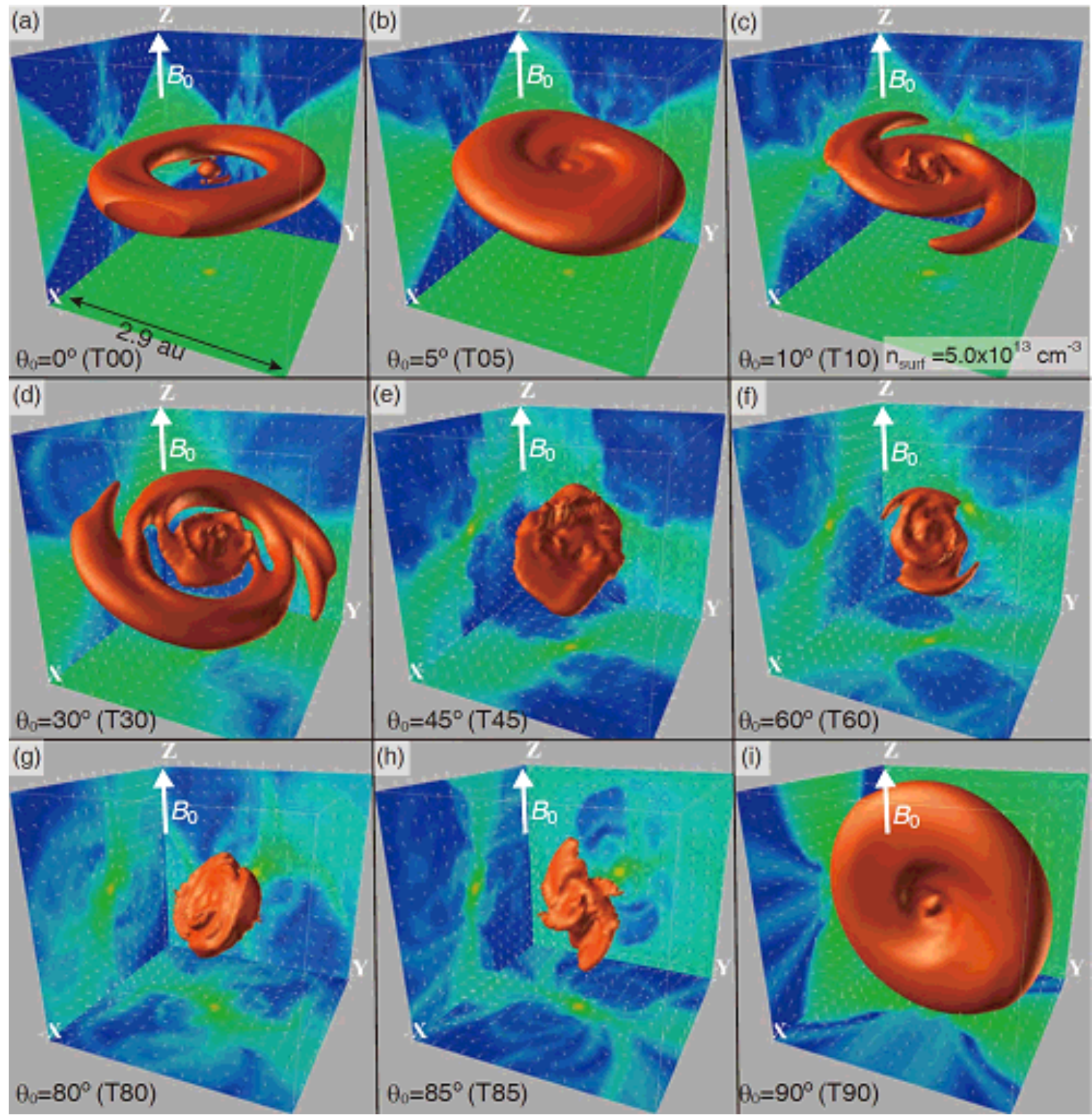

Figure 13. The iso-density surface of $5 \times 10^{13} \mathrm{~cm}^{-3}$ for all models. The box scale is 2.9 au in all the panels. The density and velocity distributions on the $x=0, y=0$ and $z=0$ planes are projected on each wall surface. The model name is noted in each panel. The initial magnetic vector is indicated by a white arrow in each panel. 

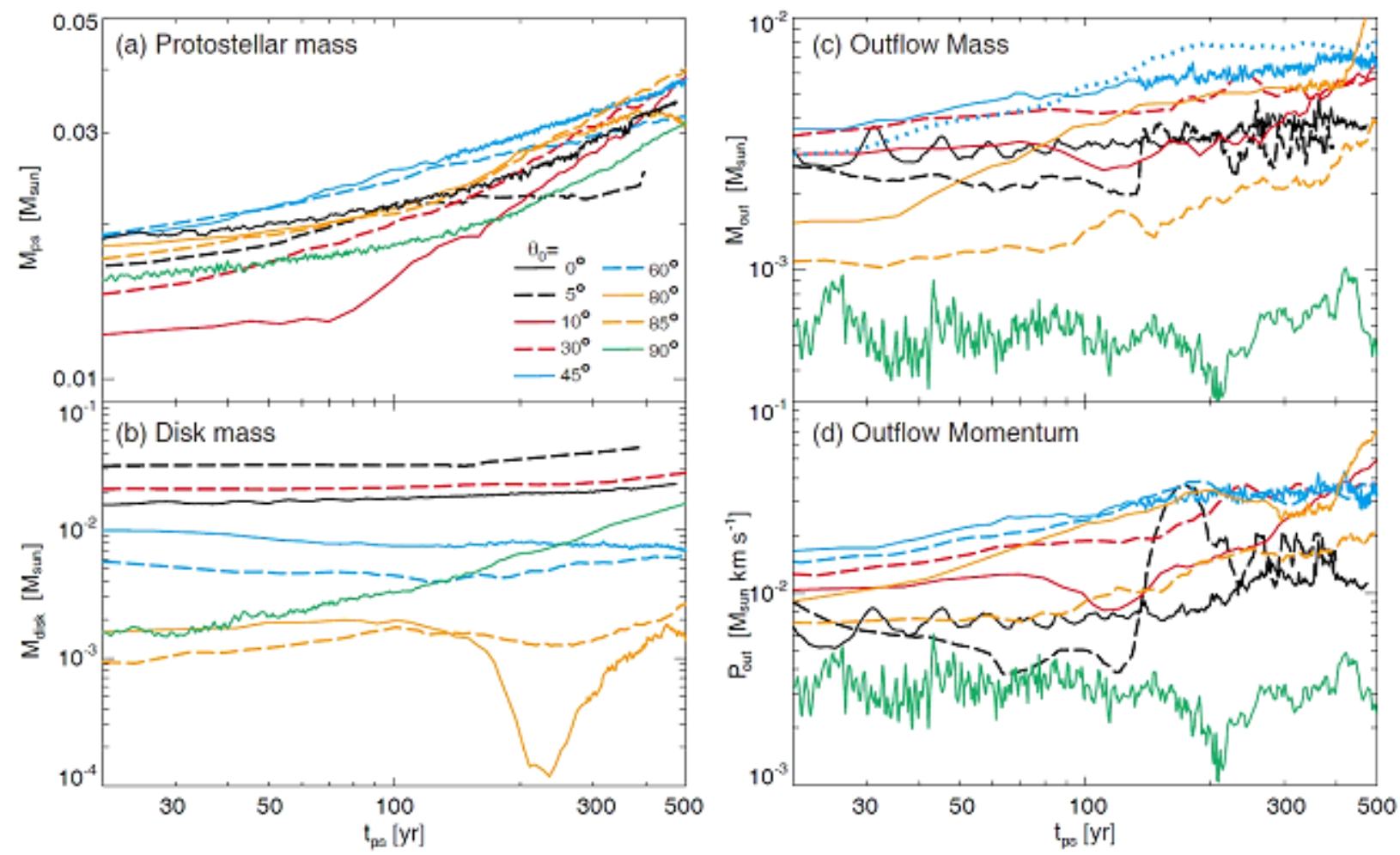

Figure 14. Protostellar mass (a), disc mass (b), outflow mass $(c)$ and outflow momentum $(d)$ for all models (T00, T05, T10, T30, T45, T60, T80, T85 and T90) against the elapsed time after protostar formation. 


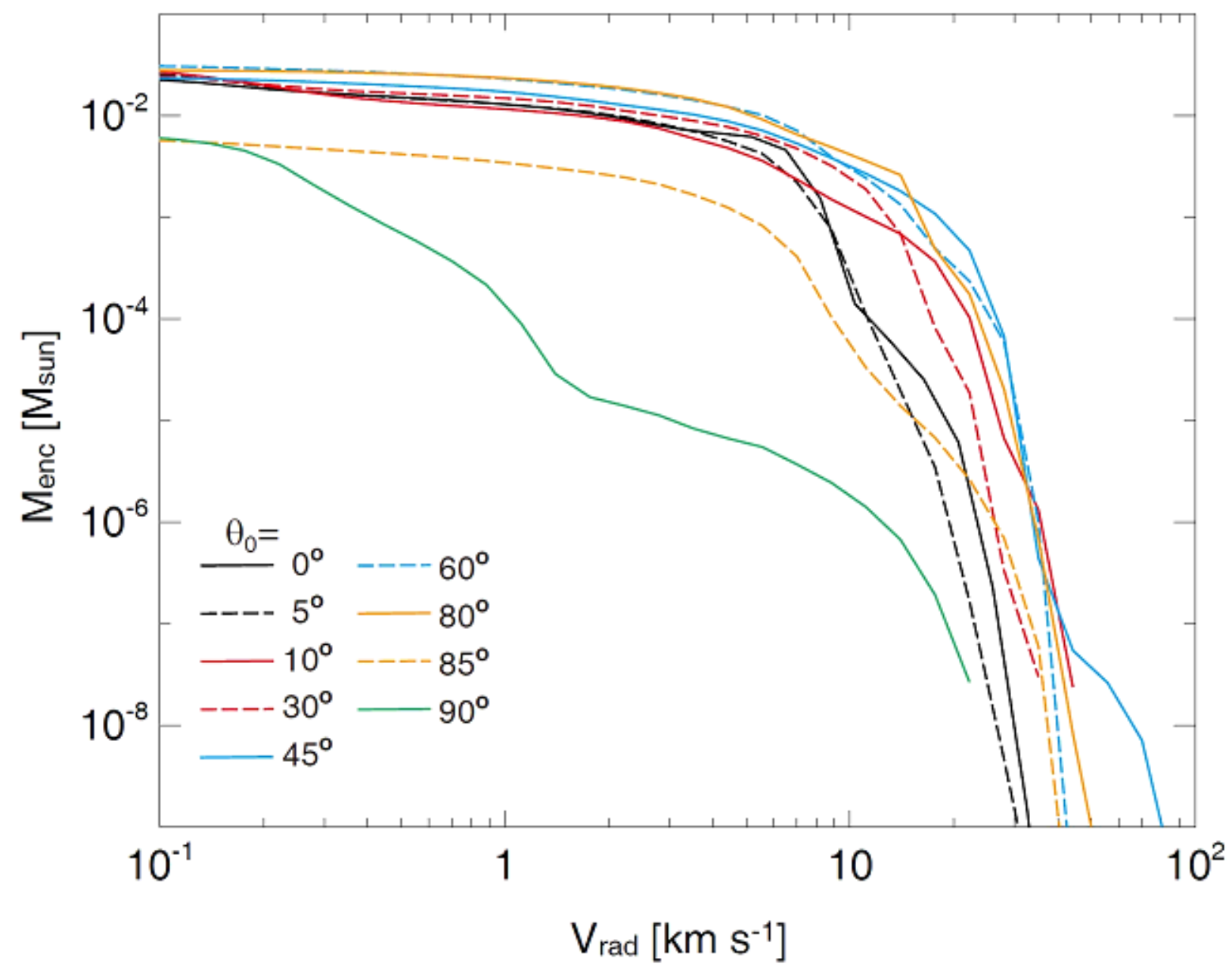

Figure 15. Outflow mass against the outflow velocity at the end of the simulation for all models. 